\title{
Sophisticated Online Learning Scheme for Green Resource Allocation in 5G Heterogeneous Cloud Radio Access Networks
}

\author{
Ismail AIQerm, Student Member, IEEE, Basem Shihada, Senior Member, IEEE
}

\begin{abstract}
G}$ is the upcoming evolution for the current cellular networks that aims at satisfying the future demand for data services. Heterogeneous cloud radio access networks (H-CRANs) are envisioned as a new trend of $5 \mathrm{G}$ that exploits the advantages of heterogeneous and cloud radio access networks to enhance spectral and energy efficiency. Remote radio heads (RRHs) are small cells utilized to provide high data rates for users with high quality of service (QoS) requirements, while high power macro base station (BS) is deployed for coverage maintenance and low QoS users service. Inter-tier interference between macro BSs and RRHs and energy efficiency are critical challenges that accompany resource allocation in H-CRANs. Therefore, we propose an efficient resource allocation scheme using online learning, which mitigates interference and maximizes energy efficiency while maintaining QoS requirements for all users. The resource allocation includes resource blocks (RBs) and power. The proposed scheme is implemented using two approaches: centralized, where the resource allocation is processed at a controller integrated with the baseband processing unit and decentralized, where macro BSs cooperate to achieve optimal resource allocation strategy. To foster the performance of such sophisticated scheme with a model free learning, we consider users' priority in RB allocation and compact state representation learning methodology to improve the speed of convergence and account for the curse of dimensionality during the learning process. The proposed scheme including both approaches is implemented using software defined radios testbed. The obtained results and simulation results confirm that the proposed resource allocation solution in H-CRANs increases the energy efficiency significantly and maintains users' QoS.
\end{abstract}

Index Terms-Resource allocation, Energy efficiency, Testbeds, Online learning, H-CRANs

\section{INTRODUCTION}

The wireless industry is witnessing an avalanche of mobile traffic fueled by the increasing interest in high data rate services such as video conferencing, online high definition video streaming, and the increasing popularity of handheld devices. 5G is envisioned as the potential solution to handle this extraordinary data rate demand [1] [2]. Cloud radio access networks (CRANs) are recognized to reduce operating expenditures, manage inter-cell interference, and provide high data rates with considerable energy efficiency performance [3] [4] [5]. It consists of remote radio heads (RRHs), which act as relays that forwards the users (UEs) data to the centralized baseband unit (BBU) pool for processing through wired/wireless fronthaul links. However, the fronthaul links limited capacity and long delays are limiting factors that degrade the performance of CRANs [6]. The energy efficiency of CRANs has been studied in the literature while designing resource allocation schemes. The authors in [7] formulated joint RRH selection and power consumption minimization, subjected to user QoS requirements and $\mathrm{RRH}$ power budget, as a group sparse beamforming problem. However, the proposed scheme ignored the fact that the fronthaul links have a limited capacity. The work in [8] aimed at optimizing the endto-end TCP throughput performance of Mobile Cloud Computing (MCC) users in a CRAN network through topology configuration and rate allocation. One limitation of this work is that it did not constrain the capacity consumption of individual links. The authors in [9] minimized the total network power consumption in a CRAN subjected to users' QoS for both secure communication and efficient wireless power transfer, limited backhaul capacity, and power budget constraints. However, this proposal considers single tier network only.

Consequently, it is necessary to decouple data and control signals in order to alleviate the influence of fronthaul links on energy efficiency and dedicate RRHs to provide high data rates without considering control functions [10]. On the other hand, high power macro base stations (BSs) in heterogeneous networks support coverage and guarantee backward compatibility with traditional cellular networks since small cells focus only on boosting the data rate in special zones [11] [12] [13]. Despite the fact that heterogeneous networks are able to improve the coverage and the capacity, inter-tier interference and the cumulative power consumption of the small cells are critical challenges that must be considered [14] [15]. The energy efficiency oriented resource allocation for heterogeneous networks attracts the researchers' attention in the literature. In [16], distributed power allocation for multi-cell OFDMA networks taking both energy efficiency and intercell interference mitigation into account was investigated, where bi-objective problem was formulated and solved using multi-objective 
optimization. The power allocation, resource block (RB) allocation and relay selection were optimized in [17] with the goal of maximizing energy efficiency. An optimal power allocation algorithm using equivalent conversion is proposed in [18] to maximize energy efficiency under interference constraints. The authors in [19] explore a system framework of cooperative green heterogeneous networks for 5G wireless communication systems. A joint efficient resource allocation was proposed in [20]. To realize the 5G vision, Heterogeneous networks with massive densification of small cells and CRANs are combined in one network structure called heterogeneous cloud radio access networks (H-CRANs) to improve spectral efficiency, resource management, and energy efficiency [21] [22].

In this paper, we propose a green resource allocation scheme for the downlink of H-CRANs between RRHs and their associated UEs that aims at maximizing energy efficiency while satisfying the UE QoS requirements and mitigate the inter-tier interference. Allocation includes $\mathrm{RB}$ assignment and power allocation. The scheme is developed using centralized and decentralized enhanced online learning approaches. The centralized allocation is performed at a designated controller integrated with the BBU, thanks to the macro BS, which is interfaced to the BBU pool for coordinating the inter-tier interference and exchange resource allocation control signals. This alleviates the capacity and time delay constraints on the fronthaul and supports the burst traffic efficiently. The decentralized approach relies on macro BSs cooperation and local information analysis to let the macro BSs make decisions to reach the appropriate allocation. The proposed learning methodology functions in conjunction with an enhanced spectrum partitioning that classifies the available RBs according to the users traffic priority and location. For example, UEs with high QoS traffic and located at the cell edge consume RBs that belongs to RBs set that is dedicated for high priority users. Moreover, compact state representation and Q-value approximation are employed to handle the curse of dimensionality during the learning process due to the large state space. To the best of our knowledge, there is no solutions in the literature for resource allocation in H-CRANs with consideration of energy efficiency using machine learning techniques. One of the advantages of online learning is that it is model-free, which facilitates its usage in dynamic heterogeneous networks.

The key contributions of this paper are summarized as follows,

- We develop an enhanced spectrum partitioning model that divides the available spectrum into two RBs sets, where each set is dedicated for certain group of users according to their location and QoS requirements.

- A centralized joint RBs and power allocation scheme for RRH and their associated UEs is proposed, which relies on a single controller integrated to the BBU. This controller acquires the state infor- mation and selects the most appropriate actions that enhance energy efficiency and guarantee QoS requirements for UEs from different tiers.

- To eliminate the single point of failure possibility and exploit the environment awareness capability of the macro BSs, we propose a decentralized resource allocation scheme, where the macro BSs utilize their local information and the BBU pool data to select the most appropriate resource allocation actions.

- The proposed online learning model incorporates compact state representation and Q-value approximation to handle the curse of dimensionality, and augment the algorithm convergence.

- We implement both resource allocation schemes using software defined radio testbed to demonstrate the schemes capability to perform efficient resource allocation in terms of energy efficiency, BS capacity and the encountered bit error rate (BER).

The paper is organized as follows, the motivation for this paper is emphasized in section 2 while section 3 presents the system model and the problem formulation. The learning model for resource allocation is described in section 4 . The approximated learning based centralized approach is illustrated in section 5 while section 6 describes the decentralized multi-agent based resource allocation methodology. Testbed implementation setup along with the achieved results are presented in section 7. Numerical results are presented in section 8 and the paper concludes in section 9 .

\section{Motivation}

H-CRANs aim at exploiting the advantages of both heterogeneous networks and CRANs and overcome their drawbacks. Comparing with CRANs and heterogeneous networks, H-CRANs have been shown to exhibit significant performance gains though advanced collaborative signal processing and radio resource allocation are still challenging. Inter-tier interference between the macro BSs and RRHs has a severe impact on energy efficiency. Unlike traditional CRANs, inter-tier interference should be controlled by an advanced processing technique and the interference to the macro UEs must be maintained at low levels with sophisticated power allocation techniques. The intra-tier interference between the RRHs is another factor to degrade energy efficiency in H-CRANs in addition to the inter-tier interference because of the fronthaul capacity constraints. In addition, the UEs usually prefer to associate with RRHs because lower transmission power is needed and more resources are allocated compared to association with macro BSs. This is reflected on energy efficiency of RRHs. Some advanced algorithms in heterogeneous networks, that consider cell association and fractional frequency reuse (FFR), have been proposed in [23] and [24], respectively to mitigate inter-tier interference and improve spectral and energy efficiency. The EE-HCRAN in [24] aims to investigate the joint optimization problem with $\mathrm{RB}$ and 
power allocation in H-CRANs under the constraint of the inter-tier interference and maintaining system capacity at certain level. The optimization problem is formulated as a non-convex problem with the goal to maximize energy efficiency. To deal with the non-convexity, an equivalent convex problem is created based on which an iterative algorithm is proposed to achieve optimal solution. In [25], a power and sub-channel allocation problem for OFDM heterogeneous networks is investigated by considering all users' rate requirements and inter-cell interference, a non-convex problem is formulated for power saving purposes. An energy efficiency oriented power and beam-forming vector allocation problem of OFDMA heterogeneous network is proposed in [26], where by decomposing main problem to multiple single constraint problems, a non-convex problem is solved. Authors in [27] proposed a joint optimization for RBs and power allocation subjected to interference constraints in $\mathrm{H}-$ CRANs. The work in [28] formulated a joint optimization problem of relay selection, power allocation and network selection to maximize the energy efficiency in H-CRANs. However, the proposed schemes are limited to the specified optimization model, which is not practical in such dynamic environment. Thus, inter-tier interference with QoS guarantee for each tier user, and resource allocation including RBs and power in an energy efficient fashion in H-CRANs is still a challenging problem that is not well explored.

\section{System Model and Problem Formula- TION}

The architecture of the considered H-CRANs system is presented in Fig. 1, in which a two-tier cellular network is shown, the macro tier and the small cells tier. The macro BSs denoted by $u \in \mathbf{U}$ are underlaid with small cells (RRHs) denoted by $s \in \mathbf{S}$, and the BBU pool performs all baseband processing functionalities. The resource allocation process for RRHs is executed at the controller integrated with the BBU pool in the centralized approach and it is achieved through cooperation between macro BSs in the distributed approach. The interRRH interference between RRHs is jointly coordinated at the BBU pool. The network users in this model are classified into two categories: MUEs denoted by index $m \in \mathbf{M}$, which are the users associated with the macro BS, while RUEs with index $n$ are the users associated with RRH. The backhaul interface is utilized to link the macro BSs with the BBU for control exchange. All the RRHs are connected to the BBU pool by the fronthaul links to facilitate data processing, transmissions, and cloud computing. The system RBs are denoted by $k \in \mathbf{K}$ with total bandwidth $B$. To enhance the spectral efficiency and mitigate the inter-tier interference, the available spectrum is partitioned into two sets of RBs: the first set denoted by $\Gamma_{1}$ incorporates RBs dedicated to RUEs with high QoS requirements or located at their corresponding cell edge. The second set of RBs denoted by $\Gamma_{2}$ comprises
RBs to be shared among RUEs with low QoS or located at the cell center and MUEs [29]. This specific partitioning improves the spectrum efficiency compared to the traditional schemes and avoids random exploration of machine learning in RBs allocation, which expedites the learning speed of convergence. The QoS requirement is defined as the minimum data rate of the RUEs. The sets $\mathbf{N}=\{1,2, \ldots ., N\}$ and $\mathbf{q}=\{N+1, \ldots . N+q\}$ represent the users associated with RRH and occupying the sets $\Gamma_{1}$ and $\Gamma_{2}$ respectively. Let us assume that $a_{n}^{k}$ is the RB allocation indicator, $a_{n}^{k}=1$, if the RB $k$ is allocated to RUE $n$ associated with RRH $s$ and 0 otherwise. For an RUE $n$ served by RRH $s$ on RB $k$, the received signal can be written as,

$$
\eta_{s, n}^{k}=P_{s, n}^{k} g_{s, n}^{k} \rho_{n}^{k}+I_{n}^{k}+N_{0}
$$

where $P_{s, n}^{k}$ is the transmission power of RRH $s$ allocated to RUE $n$ on RB $k, \rho_{n}^{k}$ is the transmitted information symbol for RUE $n$ on RB $k, N_{0}$ is the noise power, and $g_{s, n}^{k}=H_{s, n}^{k} l_{s, n}$ is the channel gain between RRH $s$ and RUE $n$ on RB $k$. Note that $H_{s, n}^{k}$ and $l_{s, n}$ refer to the fast fading coefficient and the path loss between RRH $s$ and RUE $n$ respectively. The encountered interference denoted by $I_{n}^{k}$ is calculated according to the category of the RUE $n$ determined by the set of RBs it accesses. Therefore, $I_{n}^{k}$ is calculated as follows,

$$
I_{n}^{k}=\left\{\begin{array}{l}
\sum_{i=1, i \neq n}^{N}\left(\sum_{r \in \mathbf{S}, r \neq s} P_{r, i}^{k} g_{r, i}^{k}\right) k \in \Gamma_{1} \\
\sum_{m=1}^{M} P_{u, m}^{k} g_{u, m}^{k}+\sum_{i=N, i \neq n}^{N+q}\left(\sum_{r \in \mathbf{S}, r \neq s} P_{r, i}^{k} g_{r, i}^{k}\right) \\
k \in \Gamma_{2}
\end{array}\right.
$$

where $P_{u, m}^{k}$ is the transmission power of macro BS $u$ allocated to MUE $m$ on RB $k$, and $g_{u, m}^{k}=H_{u, m}^{k} l_{u, m}$ is the channel gain between macro BS $u$ and MUE $m$ on RB $k$. Note that the index $i$ represents the other RUEs served by other RRH $r$. The signal to interference and noise ratio (SINR) for $n$th RUE occupying $k$ th RB is given by,

$$
\gamma_{s, n}^{k}=\frac{P_{s, n}^{k} g_{s, n}^{k}}{I_{n}^{k}+N_{0}}
$$

Similarly, the received signal by an MUE $m$ served by macro BS $u$ is written as follows,

$$
\eta_{u, m}^{k}=P_{u, m}^{k} g_{u, m}^{k} \rho_{m}^{k}+I_{m}^{k}+N_{0}
$$

where $\rho_{m}^{k}$ is the received information symbol and $I_{m}^{k}$ is the encountered interference by the MUE $m$ allocated to $\mathrm{RB} k$ and it is evaluated as follows,

$$
I_{m}^{k}=\sum_{i=N}^{N+q}\left(\sum_{s \in \mathbf{S}} P_{s, i}^{k} g_{s, i}^{k}\right)
$$

The SINR achieved by MUE $m$ utilizing RB $k$ and associated with macro BS $u$ is found as follows,

$$
\gamma_{u, m}^{k}=\frac{P_{u, m}^{k} g_{u, m}^{k}}{I_{m}^{k}+N_{0}}
$$




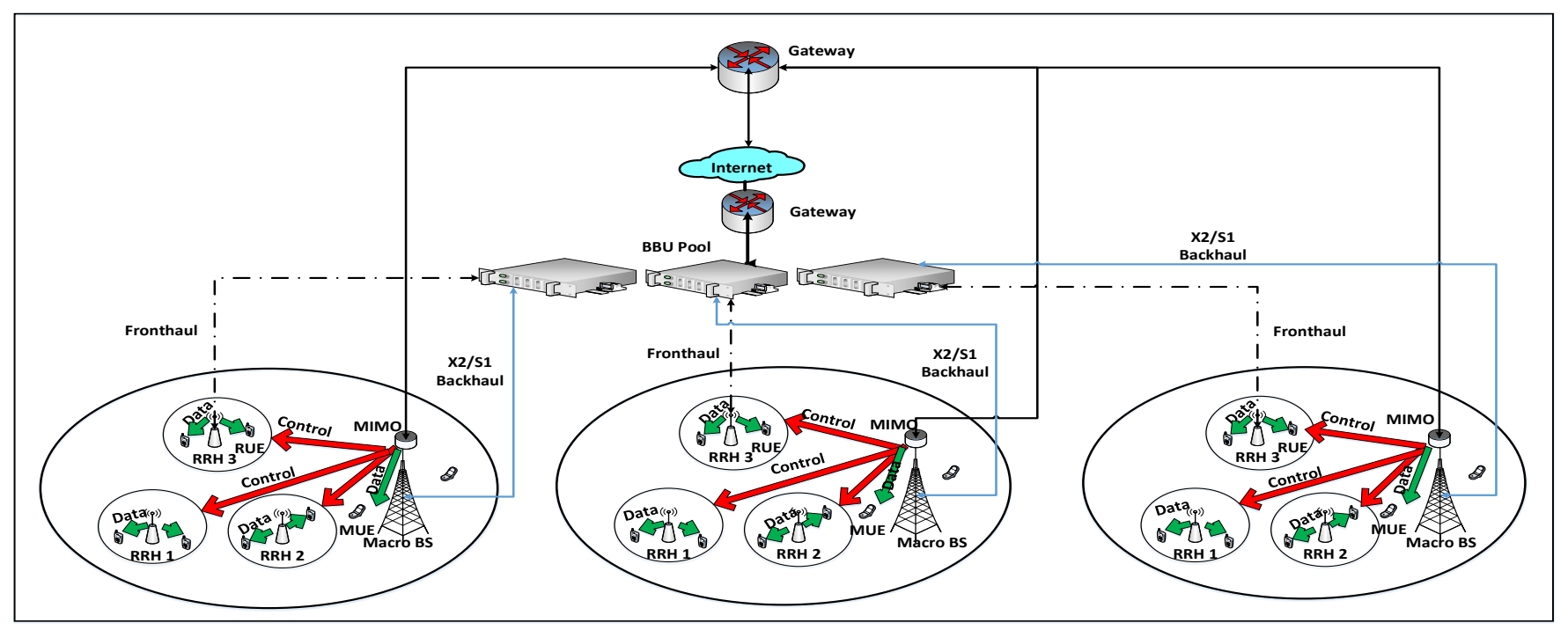

Fig. 1. H-CRANs Architecture

The capacity for all RUEs associated with RRH $s$ is expressed as follows,

$$
C_{s}=\sum_{n=1}^{N+q} \sum_{k=1}^{K} a_{s, n}^{k} B \log _{2}\left(1+\gamma_{s, n}^{k}\right)
$$

Similarly, the capacity of all MUEs associated with the macro BS $u$ is found as follows,

$$
C_{u}=\sum_{m=1}^{M} \sum_{k=1}^{K} a_{u, m}^{k} B \log _{2}\left(1+\gamma_{u, m}^{k}\right)
$$

where $a_{u, m}^{k}$ is the RB resource allocation indicator similar to $a_{s, n}^{k}$. The power consumption of the H-CRANs system is composed of the power consumed by RRHs and their related links and the power consumed by macro BSs [30]. The power consumption of RRH $s$ is evaluated as follows,

$$
P C_{s}=P_{c t}+P_{f}+P_{e}+\sum_{n=1}^{N+q} \sum_{k=1}^{K} P_{s, n}^{k} a_{s, n}^{k}
$$

where $P_{c t}$ is the circuit power, $P_{f}$ is the power consumption of the fronthaul links, and $P_{e}$ is the power consumed in signal exchange.

The energy efficiency $(E E)$ of the considered $\mathrm{H}$ CRANs system is defined as follows,

$$
E E=\frac{\mathbf{s} \times C_{s}+C_{u}}{\mathbf{S} \times P C_{s}+P C_{u}}
$$

where $P C_{u}$ is the power consumed by the macro tier. However, H-CRANs are established based on dense RRH deployment (i.e. the number of RRHs is much larger than macro BSs). The inter-tier interference from RRHs to MUEs remains constant when the density of RRHs is sufficiently high. Thus, the downlink spectral efficiency and energy efficiency performances for the macro BSs can be assumed to be stable [24] [30]. Therefore, with sufficiently high $\mathbf{S}$, the energy efficiency can be defined as,

$$
E E=\frac{C_{s}}{P C_{s}}
$$

The allocation of RBs $a_{s, n}^{k}$ and transmission power $P_{s, n}^{k}$ with objective of EE maximization in the downlink of $\mathrm{H}$ CRANs subjected to QoS requirements for all RUEs and MUEs and the fronthaul links constraints is formulated as follows,

$$
\begin{gathered}
\max _{P_{s, n}^{k}, a_{s, n}^{k}} E E \quad s . t \\
\text { C1: } \sum_{k=1}^{K} a_{s, n}^{k} \leq z_{n}, \forall s \in \mathbf{S} \\
\text { C2: } a_{s, n}^{k} \in\{0,1\} \quad \forall s \in \mathbf{S}, \quad \forall k \in \mathbf{K} \\
\text { C3: } \sum_{k=1}^{K} a_{s, n}^{k} B \log _{2}\left(1+\gamma_{s, n}^{k}\right) \geq \theta, \forall n \in \mathbf{N} \\
\text { C4: } \sum_{k=1}^{K} a_{s, n}^{k} B \log _{2}\left(1+\gamma_{s, n}^{k}\right) \geq \theta^{*}, \forall n \in \mathbf{q} \\
\text { C5: } \sum_{k=1}^{K} a_{u, m}^{k} B \log _{2}\left(1+\gamma_{u, m}^{k}\right) \geq \delta, \forall m \in \mathbf{M}
\end{gathered}
$$

C6: $P_{s, n}^{k} \leq a_{s, n}^{k} P_{s, \max }, \forall s \in \mathbf{S}, \forall n \in \mathbf{N} \cup \mathbf{q}, \forall k \in \mathbf{K}$

$$
\text { C7: } \sum_{n=1}^{N+q} \phi\left(\sum_{k=1}^{K} P_{s, n}^{k}\right) \leq \chi_{\max }, \forall s \in \mathbf{S}
$$

The constraint $\mathrm{C} 1$ limits the number of allocated RBs to RUE $n$ to $z_{n}$ RBs, which prevents the cloud from greedily allocating all the available RBs to its RUEs in order to leave some RBs for the other network tiers. C2 indicates that $a_{s, n}^{k}$ is a binary variable. The capacity constraints $\mathrm{C} 3$ and $\mathrm{C} 4$ ensure that the achieved capacity for RUE with high QoS requirements and low QoS requirements RUE is above the thresholds $\theta$ and $\theta^{*}$ respectively. C5 is the constraint to guarantee QoS for the MUE $m$. The transmit power on an unallocated RB is enforced to be zero and the maximum allowed power for each RRH is indicated in C6 as $P_{s, \max }$. Finally, C7 is a fronthaul constraint, which limits the number of baseband signals transmitted on the fronthaul link between the cloud and 
RRH $s . \chi_{\max }$ is defined as the maximum number for the transmitted signals on the fronthaul link between the cloud and RRH $s$ and $\phi$ is a step function that takes value 1 if $\sum_{k=1}^{K} P_{s, n}^{k}>0$ and 0 otherwise. $P_{s, n}^{k}<0$ indicates that RRH $s$ does not serve RUE $n$ and that the fronthaul link between the cloud and RRH $s$ does not carry the baseband signal for RUE $n$. On the other hand, if there is at least one RB $k$ such that $P_{s, n}^{k}>0$ then RRH $s$ serves RUE $n$ and the fronthaul link between the cloud and RRH $s$ is active. The resource allocation problem in (12) is tackled using enhanced online learning to reach ultimate allocation of $\mathrm{RB}$ and transmission power to guarantee capacity in the designated sub-bands and inter-tier interference mitigation.

\section{Online Learning Model for Resource ALLOCATION}

It is challenging to determine the exact state transition model by applying a model-based dynamic programing algorithm for the following reasons: The spectrum partitioning based RB allocation considered in this work is not only location dependent but also considers QoS requirements. Moreover, it is not trivial to list all the states and action pairs to migrate from one state to another, and it is not practical to predefine the state transition model in problem solving. For these reasons, we choose online Q-learning [31] for resource allocation in the H-CRANs specified model. The basic concept of online learning is described as follows, when the network is in state $x^{t}$ at time step $t$, a finite number of possible actions $y^{t}$, which are elements of the action space $Y$ that can be selected. As a result, a reward is received, which is the network feedback for the action selected at state $x^{t}$. In this section, we describe the learning model employed to achieve resource allocation that maximizes the network energy efficiency. We suppose that the resource allocation action is chosen by the network controller in the centralized approach and it is a result of the cooperative decision made by the macro BSs in the distributed one within a slotted time step $t$. The considered learning model for resource allocation is defined as $\zeta=\left(\mathbf{N}, \mathbf{q}, \gamma_{s, n}^{k}, \gamma_{u, m}^{k}, a_{s, n}^{k}, P_{s, n}^{k}, E E\right)$.

The online learning parameters are defined as follows:

- State: the environment state at certain time step $t$ is defined as, $x_{n}^{t}=\left(n\right.$, RUE location, $\left.\theta, \theta^{*}, \delta, \gamma_{s, n}^{k}, \gamma_{u, m}^{k}\right)$. The state information is acquired from the BBU and from the macro BSs that are assumed to be aware of the small cells operate under their coverage.

- Action: the action $y_{n}^{t}=\left(a_{s, n}^{k}, P_{s, n}^{k}\right)$ is defined as the allocation of RB and transmission power, RRH allocated to its associated RUEs.

- Reward: the reward function is the energy efficiency and is defined as,

$$
R(x, y)=E E(x, y)
$$

This indicates that the reward is achieved if the conditions in $\mathrm{C} 1$ to $\mathrm{C} 7$ are satisfied.
- Transition Function: for a given resource allocation strategy $\pi \in \Pi$, the state transition probability is defined as follows,

$$
T\left(x, y, x^{\prime}\right)=\operatorname{Pr}\left(x(t+1)=\left.x^{\prime}\right|^{t}=x, y^{t}=y\right)
$$

Basically, the strategy $\pi$ is defined as the probability of selection of action $y$ at state $x$.

The optimal Q-value of the online learning model is defined as the current expected reward plus a future discounted reward as follows,

$$
\begin{gathered}
Q^{*}(x, y)=E[E E(x, y)] \\
+\beta \sum_{s^{\prime} \in X} T_{x, x^{\prime}}(y) \max _{y^{\prime} \in Y} Q^{*}\left(x^{\prime}, y^{\prime}\right)
\end{gathered}
$$

where $T_{x, x^{\prime}}($.$) is the state transition probability and \beta$ is the discount factor. The optimal Q-value $Q_{n}^{*}(x, y)$ is learned by updating the Q-value function on the transition from state $x$ to state $x^{\prime}$ under the action $y$ in time stept $t$ as follows,

$$
\begin{aligned}
Q^{t+1}(x, y) & =\left(1-\alpha^{t}\right) Q^{t}(x, y)+\alpha^{t}[E E(x, y) \\
& \left.+\beta \max _{y^{\prime} \in Y} Q^{t}\left(x^{\prime}, y^{\prime}\right)\right]
\end{aligned}
$$

where $\alpha^{t} \in(0,1]$ is the learning rate. The initial Q-value for all $(x, y)$ is arbitrary. The considered online learning model here is a stochastic approximation method that solves the Bellman's optimality equation associated with the discrete time Markovian decision process (DTMDP). Online learning does not require explicit state transition probability model and it converges with probability one to an optimal solution if $\sum_{t=1}^{\infty} \alpha^{t}$ is infinite, $\sum_{t=1}^{\infty}\left(\alpha^{t}\right)^{2}$ is finite, and all state action pairs are visited infinitely often [32]. Balancing exploration and exploitation is an essential issue in the stochastic learning process. Exploration aims to try new allocation strategies so it does not only apply the strategies it already knows to be good but also explore new ones. Exploitation is the process of using well-established strategies. The most common technique to achieve exploration vs exploitation balance is to use the $\epsilon$-greedy selection [33], where $\epsilon$ is the percent of the time that an agent takes a randomly selected action rather than taking the action that is most likely to maximize its reward given what it knows so far. It usually starts with a lot of exploration (i.e. a higher value for $\epsilon$ ). Over time, as the agent learns more about the environment and which actions yield the most long-term reward, it steadily reduce $\epsilon$ as it settles into exploiting what it knows. However, $\epsilon$-greedy selects equally among the available actions i.e. ( the worst action is likely to be chosen as the best one). In order to overcome this drawback, the action selection probabilities are varied as a graded function of the Q-value. The best power level is given the highest selection probability, while all other levels are ranked according to their Q-values. The learning algorithm exploits Boltzmann probability distribution [34] to determine the probability of the resource allocation action that fulfills the energy efficiency 
maximization constraints in $\mathrm{C} 1$ to $\mathrm{C} 7$. Thus, the action $y$ in state $x$ is selected at $t$ with the following probability,

$$
\pi_{n}^{t}(x, y)=\frac{e^{Q^{t}(x, y) / \tau}}{\sum_{y^{\prime} \in Y} e^{Q^{t}\left(x, y^{\prime}\right) / \tau}}
$$

where $\tau$ is a positive integer that controls the selection probability. With high value of $\tau$, the action probabilities become nearly equal. However, low value of $\tau$ causes big difference in selection probabilities for actions with different Q-values. One issue to report is that the 5G H-CRANs system has a large space. Therefore, the curse of dimensionality increases the required computations and makes it unfeasible to use the typical online learning methodology to maintain the Q-value for each state/action pair, which slows the system convergence.

\section{Centralized Approximated Online Learning Resource Allocation Scheme}

In this approach, the resource allocation process is performed at a dedicated controller that is integrated with the BBU pool and the macro BSs act as brokers between the controller and the RRHs for control exchange with the goal of inter-tier interference mitigation and energy efficiency maximization. Resource allocation and data processing signals from the controller to the macro BSs are sent through $\mathrm{X} 2$ and $\mathrm{S} 1$ interfaces respectively, which are obtained from definitions of the Third-Generation Partnership Project (3GPP) standards [35]. All the entities in the network including RUEs, MUEs, RRHs report the channel state information and RUEs location to the macro BS that they operate under its coverage in a hierarchical manner. The channel state information includes path loss and channel gains from the serving RRH and the macro BS to the RUEs and MUEs. All the macro BSs provide this information to the controller through the control exchange interface. The controller exploits the reported information and QoS requirements to select the proper RB and transmission power using online learning. The allocation decision made by the controller is sent to each RRH through the macro BS. Note that SINR is the state information exploited in action selection and it is determined according to the channel information reported and the allocated transmission power.

The computational complexity of the system increases along with the size of the states and action spaces. The simple look-up table where separate Q-value is maintained for each state/action pair is not feasible in large space with massive number of states like our system. Therefore, we propose a brief representation for the Q-values in which they are approximated as a function of much smaller set of variables to account for the curse of dimensionality. The brief representation of Q-value focuses on a countable state space $X^{*}$ using the function $Q^{\prime}: X^{*} \times Y$, which is referred as a function approximator. The parameter vector $\xi=\left\{\xi_{z}\right\}_{z=1}^{Z}$ is adopted to approximate the Q-value by minimizing the metric of difference between $Q^{*}(x, y)$ and $Q^{\prime}(x, y, \xi)$ for all $(x, y) \in X^{*} \times Y$. Thus, the approximated $Q^{\prime}$ value is formalized as follows,

$$
Q^{\prime}(x, y, \xi)=\sum_{z=1}^{Z} \xi_{z} \psi_{z}(x, y)=\xi \boldsymbol{\psi}^{\boldsymbol{T}}(x, y)
$$

where $T$ denotes the transpose operator and the vector $\psi(x, y)=\left[\psi_{z}(x, y)_{z=1}^{Z}\right]$ with a scalar function $\psi_{z}(x, y)$ defined as the basis function (BF) over $X^{*} \times Y$, and $\xi_{z}(z=1, \ldots, Z)$ are the associated weights. A gradient function $\psi(x, y)$, which is a vector of partial derivative with respect to the elements of $\xi^{t}$, is used to combine the typical online learning model defined in (16) with the linearly parametrized approximated online learning proposed.

The Q-value update rule in (16) is reconstructed to include the parameter vector updates as follows,

$$
\begin{gathered}
\xi^{t+1} \boldsymbol{\psi}^{\boldsymbol{T}}(x, y)=\left\{\left(1-\alpha^{t}\right) \xi^{t} \psi^{T}(x, y)+\right. \\
\left.\alpha^{t}\left[E E(x, y)+\beta \max _{y^{\prime} \in Y} \xi^{t} \boldsymbol{\psi}^{\boldsymbol{T}}\left(x^{\prime}, y^{\prime}\right)\right]\right\} \psi(x, y)
\end{gathered}
$$

The probability of selecting certain action presented in (17) is updated with the Q-value approximation as follows,

$$
\pi^{t}(x, y)=\frac{e^{\xi^{t} \boldsymbol{\psi}^{T}(x, y) / \tau}}{\sum_{y^{\prime} \in Y} e^{\xi^{t} \boldsymbol{\psi}^{T}(x, y) / \tau}}
$$

The online learning process with approximated Q-value is illustrated in Algorithm 1. The algorithm takes the QoS

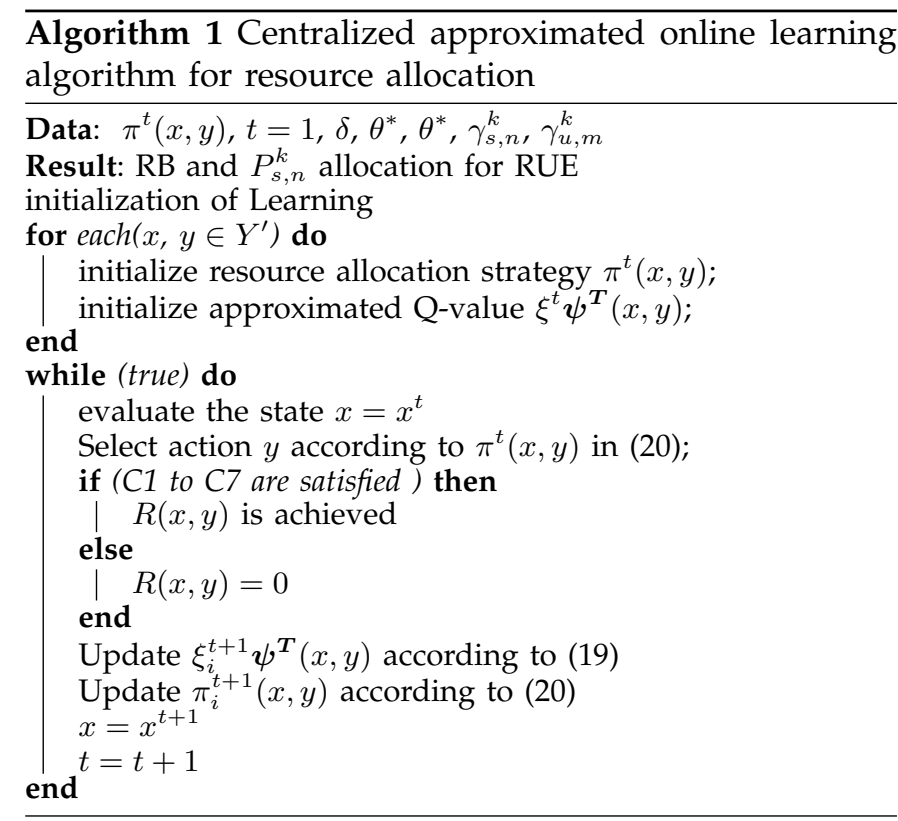

requirements for RUEs and MUEs as input to check the quality of the strategies selected and they are compared to the capacity achieved by different BSs. The algorithm selects action strategies according to (20). If the conditions $\mathrm{C} 1$ to $\mathrm{C} 7$ are satisfied, then, the reward is achieved. Finally, the Q-value and resource allocation strategy are updated according to (19) and (20) respectively, and the new state is observed. 
To demonstrate Algorithm 1 convergence, we found the necessary conditions for convergence of the proposed approximated learning resource allocation. To start the proof, we introduce the following definition and assumptions [36] [37].

Definition 1: Let $\Psi=E\left[\boldsymbol{\psi}^{\boldsymbol{T}}(x, y) \psi(x, y)\right]$. For the parameter vector $\xi$ and a particular network state $x \in X^{*}$, we define a vector $\psi(x, \xi)=\left[\psi_{z}(x, y)\right]$ for $z=1 \rightarrow Z$ where $y \in \aleph=\left\{y=\arg \max _{y^{\prime} \in Y} \xi \boldsymbol{\psi}^{\boldsymbol{T}}\left(x, y^{\prime}\right)\right\}$ is the set of optimal joint resource allocation actions for $x$. We define the following a $\xi$-dependent matrix:

$$
\Psi^{\prime}=E\left[\boldsymbol{\psi}^{\boldsymbol{T}}(x, \xi) \psi(x, \xi)\right]
$$

Assumption 1: The basis functions $\psi_{z}(x, y)$ are linearly independent for all $(x, y)$ and all the properties of $Q^{t}(x, y)$ in previous discussion are applicable to the dot product for the vectors $\xi^{t} \boldsymbol{\psi}^{\boldsymbol{T}}(x, y)$.

Assumption 2: For every $z=(1,2 \ldots . Z), \psi_{z}(x, y)$ is bounded, which means $E\left\{\psi_{z}^{2}(x, y)\right\}<\infty$ and the reward function satisfies $E\left\{R^{2}(x, y)\right\}<\infty$.

Assumption 3: The learning rate satisfies $\sum_{t=1}^{\infty} \alpha^{t}=\infty$ and $\sum_{t=1}^{\infty}\left(\alpha^{t}\right)^{2}<\infty$.

Proposition 1: With the assumptions 1-3 and Definition 1 , the approximated online learning algorithm converges with probability (w.p) 1 , if

$$
\Psi^{\prime}>\Psi, \forall \xi
$$

Proof:

The proof of convergence requires finding stable fixed points of the ordinary differential equations (ODE) associated with the update rule in (19), which can be written as.

$$
\left.\xi^{. t}=E\left[E E(x, y)+\beta \xi^{t} \boldsymbol{\psi}^{\boldsymbol{T}}\left(x^{\prime}, \xi^{t}\right)-\xi^{t} \boldsymbol{\psi}^{\boldsymbol{T}}(x, y)\right) \psi(x, y)\right]
$$

where $\xi^{t}=\frac{\partial \xi}{\partial t}$ as $\alpha \rightarrow 0$. We define two trajectories of the ODE $\xi_{1}^{t}$ and $\xi_{2}^{t}$ that have different initial conditions and satisfies $\xi_{0}^{t}=\xi_{1}^{t}-\xi_{2}^{t}$. Then, we have

$$
\begin{aligned}
\frac{\partial\left\|\xi_{0}^{t}\right\|^{2}}{\partial t}= & 2\left(\xi_{1}^{t}-\xi_{2}^{t}\right)\left(\xi_{0}^{t}\right)^{T}=2 \beta E\left[\xi_{1}^{t} \boldsymbol{\psi}^{\boldsymbol{T}}\left(x^{\prime}, \xi_{1}^{t}\right) \psi(x, y)\left(\xi_{0}^{t}\right)^{T}\right. \\
& \left.-\xi_{2}^{t} \boldsymbol{\psi}^{\boldsymbol{T}}\left(x^{\prime}, \xi_{2}^{t}\right) \psi(x, y)\left(\xi_{0}^{t}\right)^{T}\right]-2 \xi_{0}^{t} \Psi\left(\xi_{0}^{t}\right)^{T}
\end{aligned}
$$

From the definition of $\psi(x, \xi)$ in Definition 1, we can deduce the following two inequalities,

$$
\begin{aligned}
& \xi_{1}^{t} \boldsymbol{\psi}^{\boldsymbol{T}}\left(x^{\prime}, \xi_{1}^{t}\right) \geq \xi_{1}^{t} \boldsymbol{\psi}^{\boldsymbol{T}}\left(x^{\prime}, \xi_{2}^{t}\right) \\
& \xi_{2}^{t} \boldsymbol{\psi}^{\boldsymbol{T}}\left(x^{\prime}, \xi_{2}^{t}\right) \geq \xi_{2}^{t} \boldsymbol{\psi}^{\boldsymbol{T}}\left(x^{\prime}, \xi_{1}^{t}\right)
\end{aligned}
$$

As the expectation $E$ in (24) is taken over different states and different actions, we can define two sets $\Lambda_{+}=$ $\left\{(x, y) \in X \times Y \mid \xi_{0}^{t} \boldsymbol{\psi}^{\boldsymbol{T}}(x, y)>0\right\}$ and $\Lambda_{-} \in X \times Y-\Lambda_{+}$. If we combine (25) and (26) in (24), we get,

$$
\begin{array}{r}
\quad \frac{\partial\left\|\xi_{0}^{t}\right\|^{2}}{\partial t} \geq 2 \beta\left(E\left[\xi_{0}^{t} \boldsymbol{\psi}^{\boldsymbol{T}}\left(x^{\prime}, \xi_{2}^{t}\right)\right) \psi(x, y)\left(\xi_{0}^{t}\right)^{T} \mid \Lambda_{+}\right] \\
\left.\left.+E\left[\xi_{0}^{t} \boldsymbol{\psi}^{\boldsymbol{T}}\left(x^{\prime}, \xi_{1}^{t}\right)\right) \psi(x, y)\left(\xi_{0}^{t}\right)^{T} \mid \Lambda_{-}\right]\right)-2 \xi_{0}^{t} \Psi\left(\xi_{0}^{t}\right)^{T}
\end{array}
$$

After the application of Holder's inequality [38] to the expectation in (27), we get,

$$
\begin{gathered}
\frac{\partial\left\|\xi_{0}^{t}\right\|^{2}}{\partial t} \geq 2 \beta\left(\sqrt{E\left[\left(\xi_{0}^{t} \boldsymbol{\psi}^{\boldsymbol{T}}\left(x^{\prime}, \xi_{2}^{t}\right)\right)^{2} \mid \Lambda_{+}\right]} \times\right. \\
\sqrt{E\left[\left(\psi(x, y)\left(\xi_{0}^{t}\right)^{T}\right)^{2} \mid \Lambda_{+}\right]}+\sqrt{E\left[\left(\xi_{0}^{t} \boldsymbol{\psi}^{\boldsymbol{T}}\left(x^{\prime}, \xi_{1}^{t}\right)\right)^{2} \mid \Lambda_{-}\right]} \\
\left.\times \sqrt{E\left[\left(\psi(x, y)\left(\xi_{0}^{t}\right)^{T}\right)^{2} \mid \Lambda_{-}\right]}\right)-2 \xi_{0}^{t} \Psi\left(\xi_{0}^{t}\right)^{T} \\
\geq 2 \beta\left(\sqrt{E\left[\left(\xi_{0}^{t} \boldsymbol{\psi}^{\boldsymbol{T}}\left(x^{\prime}, \xi_{2}^{t}\right)\right)^{2}\right]} \times \sqrt{E\left[\left(\psi(x, y)\left(\xi_{0}^{t}\right)^{T}\right)^{2} \mid \Lambda_{+}\right]}\right. \\
\left.+\sqrt{E\left[\left(\xi_{0}^{t} \boldsymbol{\psi}^{\boldsymbol{T}}\left(x^{\prime}, \xi_{1}^{t}\right)\right)^{2}\right]} \times \sqrt{E\left[\left(\psi(x, y)\left(\xi_{0}^{t}\right)^{T}\right)^{2} \mid \Lambda_{-}\right]}\right) \\
-2 \xi_{0}^{t} \Psi\left(\xi_{0}^{t}\right)^{T}
\end{gathered}
$$

If we apply the definition of $\Psi^{\prime}$ in Definition 1, we get,

$$
\begin{aligned}
& \geq 2 \beta \sqrt{\max \left[\xi_{0}^{t} \Psi_{1}^{\prime}\left(\xi_{0}^{t}\right)^{T}, \xi_{0}^{t} \Psi_{2}^{\prime}\left(\xi_{0}^{t}\right)^{T}\right]} \\
& \times \sqrt{E\left[\left(\psi(x, y)\left(\xi_{0}^{t}\right)^{T}\right)^{2}\right]}+-2 \xi_{0}^{t} \Psi\left(\xi_{0}^{t}\right)^{T}
\end{aligned}
$$

According to the condition in (21), we can state that,

$\frac{\partial\left\|\xi_{0}^{t}\right\|^{2}}{\partial t} \geq 2 \beta \xi_{0}^{t} \Psi\left(\xi_{0}^{t}\right)^{T}-2 \xi_{0}^{t} \Psi\left(\xi_{0}^{t}\right)^{T}=(2 \beta-2) \xi_{0}^{t} \Psi\left(\xi_{0}^{t}\right)^{T}<0$

which means that $\xi_{0}^{t}$ converges to the origin and this confirms that there exists a stable point of the ODE in (23). Thus, the proposed online learning with $Q$ approximation converges w.p 1.

Consequently, the stable point $\xi^{*}$ of the ODE in (23) indicates that,

$$
\left.0=E\left[E E(x, y)+\beta \xi^{t} \boldsymbol{\psi}^{\boldsymbol{T}}\left(x^{\prime}, \xi^{t}\right)-\xi^{t} \boldsymbol{\psi}^{\boldsymbol{T}}(x, y)\right) \psi(x, y)\right]
$$

and $\xi^{*}$ can be found as follows,

$$
\left.\xi^{*}=E\left[E E(x, y)+\beta \xi^{*} \psi^{T}\left(x^{\prime}, \xi^{*}\right)\right) \psi(x, y)\right] \Psi^{-1}
$$

As a result, the approximated online Q-function is stated as follows,

$$
Q^{\prime}\left(x, y, \xi^{*}\right)=\xi^{*} \psi(x, y)
$$

\section{Decentralized Multi-Agent Online LeARning Resource Allocation Scheme}

Even with the Q-function approximation using compact representation, the number of actions can grow exponentially with the number of cells deployed in the network. Thus, to have a centralized controller for resource allocation is not the best practice. As the macro BSs and can locally manage their operations, there is a possibility to deploy a decentralized resource allocation scheme using online learning that functions through the macro BSs. All the macro BSs can learn in a cooperative manner how to make local decisions for resource allocation for the RUEs associated with RRHs. In this way, the resource allocation task is achieved using multiagent online learning, where macro BSs represent the 
agents. The macro BSs estimate the SINR according to the channel state information reported by the network nodes and the power allocated, and execute the online learning algorithm for resource allocation.

We assume that the macro BSs learn in a team Markov game defined as $G E=\left\{\mathbf{U}, X^{\prime}, Y, T, R\right\}$ with the common goal to find a joint resource allocation strategy $\pi$ that mitigates the inter-tier interference and maximizes energy efficiency, where $\mathbf{U}$ is the set of the macro BSs. The optimal Q-value $Q^{*}(x, y)$ for all $(x, y) \in X^{\prime} \times Y$, defines the optimal resource allocation strategy and capture the markovian game structure. For each network state $x \in$ $X^{\prime}$, the action in the team Markov game is generated by the $\mathbf{U}$ independent macro BSs in a decentralized fashion. The decided action $y$ at state $x$ is considered optimal if $Q^{*}(x, y) \geq Q^{*}\left(x, y^{\prime}\right)$ for all $y^{\prime} \in Y$. The macro BSs are assumed to learn using the compact representation model stated in (19). Consequently, we can deduce the following proposition.

Proposition 2: For the Markovian game $G E$, the decentralized multi-agent online learning algorithm converges w.p. 1 if the condition in Proposition 1 holds.

Proof: If we consider each macro BS as a single controller that follow a stationary resource allocation strategy, then the Markovian game is a DTMDP as in section 5. As a result, the proof follows the same procedure of the proof of Proposition 1.

To proceed with the multi-agent learning, the following assumptions are made,

Assumption 4: The resource allocation strategy of different macro BSs do not alter significantly in similar network states.

Assumption 5: The initial network state $x^{t}$ evolves following Harris recurrent Markov chain [39].

According to Assumption 4, each macro BS can conjecture the allocation strategy of other macro BSs without explicit cooperation through the use of historical knowledge if it encounters the same network state. The similarity between network states can be measured in terms of Hamming distance [40] denoted by $D H\left(x, x^{\prime}\right)$. We define the historical knowledge up to time step $t$ using $\sigma$-algebra as follows,

$$
F(t)=\sigma\left(\{x(b), y(b)\}_{b=1}^{t},\{R(x(b), y(b))\}_{b=1}^{t-1}\right)
$$

where the information of each experienced network state $x(b)$, each performed joint action $y(b)$ and network energy efficiency (the reward) $R(x(b), y(b))$ can be obtained from the BBU. At each time step $t$, each macro BS checks the Hamming distance between the current state $x(t)$ and state $x(b)$ in $F(t)$. Then, it creates a sample set $X_{F}\left(x^{t}, F(t)\right)$, which includes $F$ most recent observations from $F(t)$ that has a minimum value of $\sum_{f=1}^{F} D H\left(x^{t}, x^{b}\right)$. Now, the common reward $R_{c}(x, y)$ that all macro BSs receive after they perform a joint resource allocation action, which is defined as $y \in Y$, is set to 1 if $y=\operatorname{argmax}_{y^{\prime} \in Y} Q^{\prime}\left(x, y^{\prime}, \xi^{*}\right)$ and 0 otherwise. Moreover, we define $Y_{u}$ for each macro BS as the set of joint actions that gives the reward 1 in state $x^{t}$. The decentralized multi-agent learning for resource allocation process in H-CRANs is illustrated in Algorithm 2.

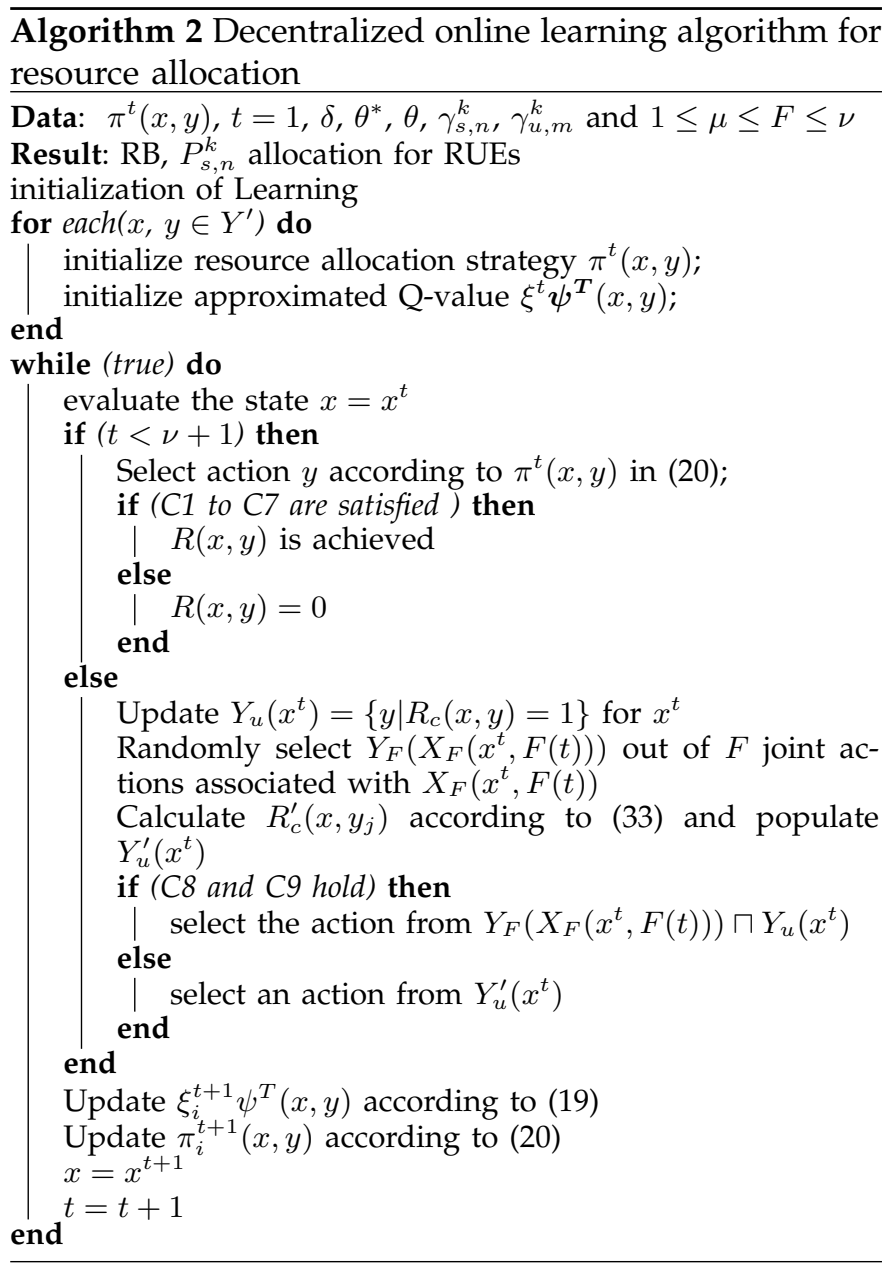

Note that $\mu$ and $\nu$ are two integers that satisfy $1 \leq$ $\mu \leq F \leq \nu$. The algorithm states that when $t<\nu+1$, the resource allocation process according to the probability in (20) is engaged to determine the resource allocation strategy. From $t=\nu+1$, each macro BS selects $\mu$ records of $Y_{F}\left(X_{F}\left(x^{t}, F(t)\right)\right)$ from the $F$ joints actions for $X_{F}\left(x^{t}, F(t)\right)$. If the following conditions C8 and C9 are met, the macro BS $u$ selects the allocation action $y_{u}\left(b^{*}\right)$, where $y_{u}\left(b^{*}\right)=\max _{b}\left\{b \mid y(b) \in Y_{F}\left(X_{F}\left(x^{t}, F(t)\right)\right) \sqcap\right.$ $\left.Y_{u}\left(x^{t}\right)\right\}$.

- C8: there exists allocation action $y=\left(y_{u}, y_{-u}\right) \in$ $Y_{u}\left(x^{t}\right)$ such that $y_{-u}^{\prime}=y_{-u} \forall y^{\prime}=\left(y_{u}^{\prime}, y_{-u}^{\prime}\right) \in$ $Y_{F}\left(X_{F}\left(x^{t}, F(t)\right)\right)$

- C9: there exists at least one action $y \in Y_{F}\left(X_{F}\left(x^{t}, F(t)\right)\right) \sqcap Y_{u}\left(x^{t}\right)$

However, if C8 and C9 are not met, macro BS $u$ will select an action from $Y_{u}^{\prime}\left(x^{t}\right)=\left\{y_{u} \mid y_{u}=\arg \max _{y_{u}^{\prime}} R_{c}^{\prime}\left(x^{t}, y_{u}^{\prime}\right)\right\}$, where

$$
R_{c}^{\prime}\left(x^{t}, y_{u}\right)=\sum_{y_{-u}} R_{c}\left(x^{t}, y\right) \frac{T_{u}^{t}\left(x^{t}, y_{-u}\right)}{\nu}
$$

$\nu$ are cases randomly drawn from $F$ most recent actions, $T_{u}^{t}\left(x^{t}, y_{-u}\right)$ is the number of times the conjectured action 
of other macro BS $y_{-u}$ in state $x^{t}$ is performed.

The convergence of $\left\{\xi^{t}\right\}$ to the optimal $\xi^{*}$ arises as a result of Proposition 2. With Assumptions 4 and 5, the team stage game $G E$ is reduced to a team game under network states $X_{F}\left(x^{t}, F(t)\right)$. According to theorem 1 in [41], the U macro BSs coordinate resource allocation strategy for all $x^{t}$ as long as $\nu \leq F /\left(\rho_{G E}+2\right)$, where $\rho_{G E}$ is the length of the shortest path in the best response graph of team stage game $G E$ [42]. This confirms that the decentralized resource allocation algorithm will converge with probability one.

\section{Testbed IMPLEMENTATION}

The H-CRANs system is implemented using radio frontends testbeds to demonstrate the performance of the proposed resource allocation scheme. The logical architecture of the considered H-CRANs in testbed implementation for both centralized and decentralized resource allocation consists of four main components: centralized BBU pool for baseband processing, macro BSs, RRHs represented by low power pico BSs, and the resource allocation controller integrated to the BBU pool. The heterogeneous network including macro and pico BSs are connected to the BBU pool through wired links.

As SINR is utilized by the online learning model for state information, this creates a need for a communication protocol that facilitates the acquisition of channel state information. The communication protocol performs three tasks: synchronization, SINR estimation and resource allocation. Therefore, the resource allocation frame structure is divided into three stages:

- Synchronization Stage: in this stage each macro BS performs synchronization with the BSs operating under its coverage through broadcasting a beacon frame periodically to compensate for any misalignment in clock frequencies of each node. Moreover, the beacon frame includes some information about the network such as the number of time steps in each stage, and the time step occupancy in the following stages.

- Acquisition Stage: this stage is a TDMA-based stage, where each UE report its channel state information to its corresponding pico BS or macro BS. It is composed of three time slots, where the first and second slots are spared for the macro BS and its associated MUEs to acquire their channel state information to find the channel gains of all links for each RB. The third slot is dedicated for pico BSs to report their channel state information while other pico BSs estimate the channel gain with that pico BS on the same RB.

- Resource Allocation Stage: at this stage, the online learning-based resource allocation algorithms are executed whether at the controller as in the centralized approach or through macro BSs in the decentralized approach.

\subsection{Implementation Setup}

We exploit GNU radio [43] as an software defined radio (SDR) development platform to create digital signals. GNU is an open-source software development toolkit that provides signal processing blocks to implement wireless protocols. As GNU radio can only handle digital data, RF front ends are required to shift the baseband signal to the desired center frequency. USRP-N210 from Ettus Research [44] is utilized as the RF front end in our setup with $\mathrm{CBX}$ daughter board that can operate in frequency range of $1 \mathrm{GHz}$ to $6 \mathrm{GHz}$ with one antenna. The USRP N210 provides high-bandwidth, highdynamic range processing capability. It is designed for demanding communications applications requiring this type of rapid development. The product architecture comprises a Xilinx Spartan 3A-DSP 3400 FPGA, 100 MS/s dual ADC, $400 \mathrm{MS} / \mathrm{s}$ dual DAC and Gigabit Ethernet connectivity to stream data to and from host processors. In addition, we add two Dell servers as SDR processors that run GNU radio to perform the baseband processing and include the controller implementation for resource allocation. The centralized and the decentralized resource allocation topologies considered in the testbed implementation are presented in Fig. 2 and Fig. 3 respectively. We have two macro BSs, four pico BSs, four RUEs, and two MUEs, where each of them is represented by one USRP-N210. In the centralized resource allocation approach, only one dell server is utilized as the controller and the unit for baseband processing. However, two servers are used, where each one processes the resource allocation algorithm implemented in each macro BS in the distributed resource allocation approach.

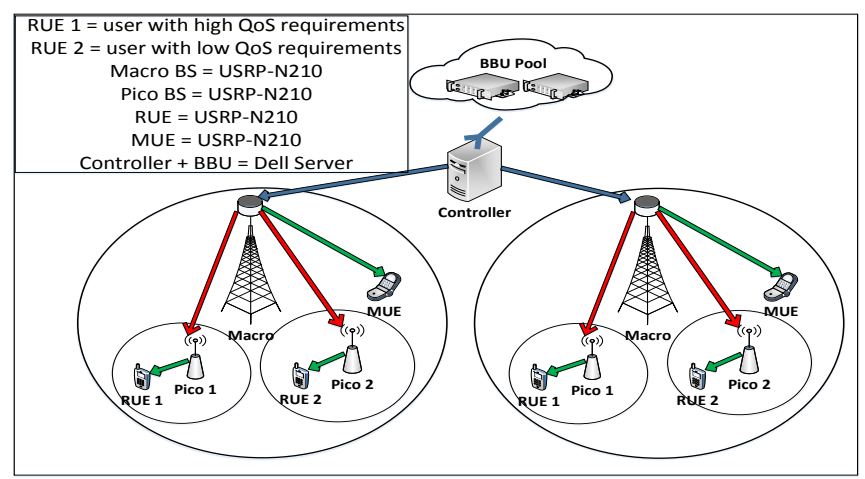

Fig. 2. Centralized testbed implementation topology

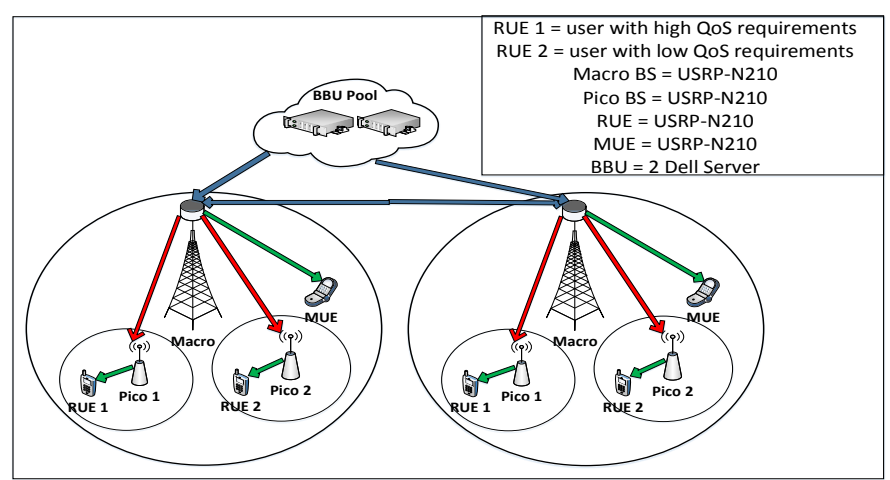

Fig. 3. Decentralized testbed implementation topology 
The conceptual models for each node in the network for both centralized and decentralized approaches for resource allocations are presented in Fig. 4. This model consists of the following modules: communication protocol module, which is common in all nodes and it performs the tasks mentioned before. The online learning algorithm module, which includes the implementation of the resource allocation process in Algorithm 1 and 2. This module is implemented in the controller in the centralized approach and at the macro BS in the distributed methodology. GNU Radio PHY module performs the physical layer functionality supported by the physical layer modules in the GNU Radio software. Finally, SINR estimation module, which relies on the probe block in GNU Radio, where the probe performs an average magnitude square process on the samples acquired during the sensing time. The acquired results from the probe block is further used to estimate the signal and the noise power. Signal power estimation is performed by averaging the acquired samples from the probe block over certain time while the noise power is estimated by considering the variance of the acquired samples. Table 1 presents the parameters used for testbed

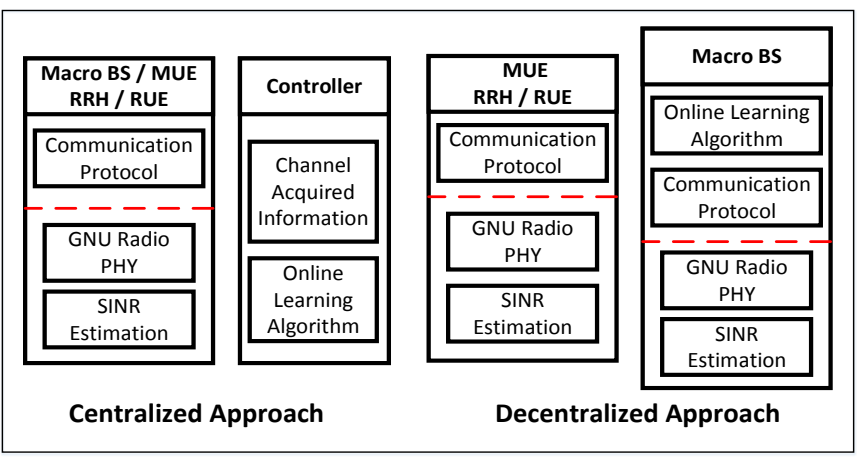

Fig. 4. Conceptual model of the resource allocation system

implementation including the communication protocol, configuration and online learning parameters.

\begin{tabular}{|c|c|}
\hline Parameter & Value \\
\hline \multicolumn{2}{|c|}{ Communication protocol parameters } \\
\hline Acquisition stage time slots & 3 \\
\hline $\begin{array}{l}\text { Sensing duration } \\
\text { modulation scheme }\end{array}$ & $\begin{array}{l}10 \mathrm{~ms} \\
\mathrm{gmsk}\end{array}$ \\
\hline \multicolumn{2}{|c|}{ Configuration Parameters } \\
\hline Macro BS Tx power & $20 \mathrm{dBm}$ \\
\hline System bandwidth & $10 \mathrm{MHz}$ \\
\hline Carrier frequency & $2.4 \mathrm{GHz}$ \\
\hline Sampling rate & $10 \mathrm{MHz}$ \\
\hline Antenna gain & $3 \mathrm{dBi}$ \\
\hline \multicolumn{2}{|c|}{ Online Learning Related Parameters } \\
\hline Learning rate $\alpha$ & 0.5 \\
\hline Discount factor $\beta$ & 0.9 \\
\hline
\end{tabular}

\subsection{Testbed Experimental Results}

In this section, we present the performance evaluation of our proposed centralized and decentralized approaches compared to the equal power allocation standard scheme. The logical structures in Fig. 2 and Fig.
3 are exploited as the network setup, where we have H-CRANs that incorporate two macro BSs. Each macro BS has two small BSs operate under its coverage and one MUE. One of the small cells communicates with an RUE 1 with high QoS requirements while the other cell is connected to low QoS RUE 2. RUE 1 traffic type is video file streaming while RUE 2 and MUE receive a small TCP file. The considered evaluation of the network operation targets UEs achieved capacity, network energy efficiency and UEs BER. Fig. 5, Fig. 6, and Fig. 7 present the average capacity achieved by the small cell with high QoS requirements RUE, the small cell with low QoS requirements RUE, and the macro BS with one MUE respectively. We notice that the our proposed scheme in

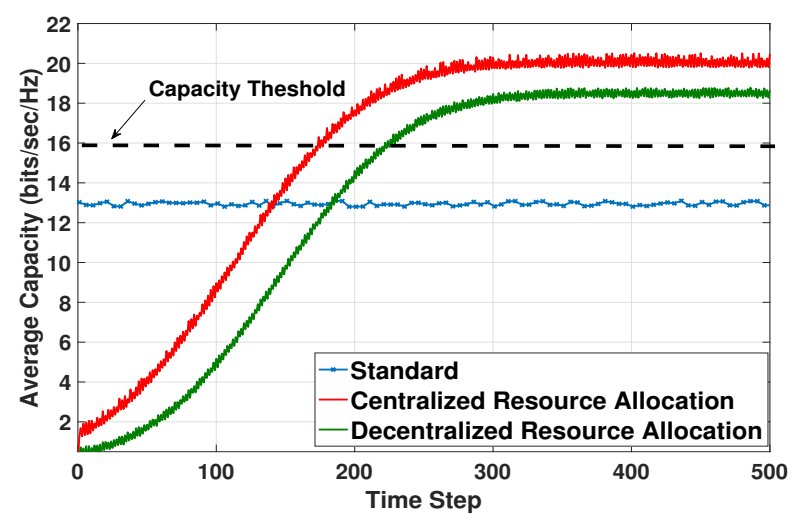

Fig. 5. Average high QoS pico BS capacity

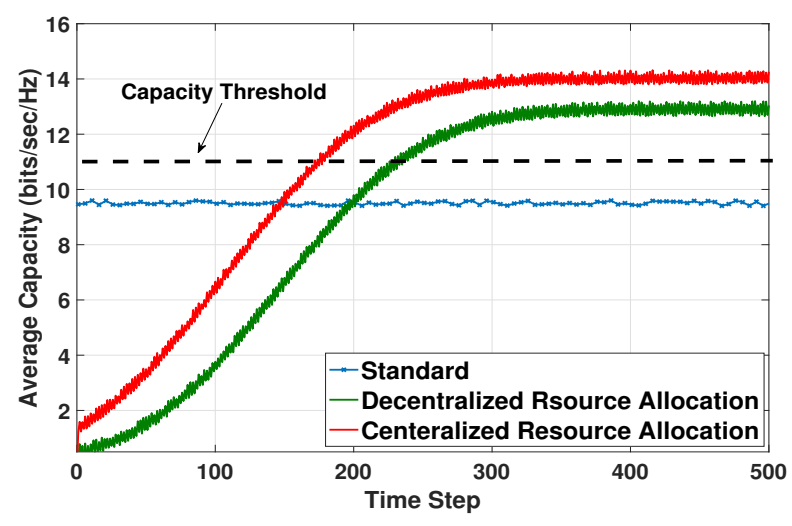

Fig. 6. Average low QoS pico BS capacity

both approaches outperforms the standard, where fixed power allocation is assumed in the achieved capacities for all users. Moreover, the proposed scheme maintains the capacity above the threshold. In Fig. 5, the centralized approach for resource allocation converges to capacity of $20 \mathrm{bits} / \mathrm{sec} / \mathrm{Hz}$ while the decentralized approach saturates at $18.5 \mathrm{bits} / \mathrm{sec} / \mathrm{Hz}$. However, the capacity of the low QoS requirements RUE is 14 bits $/ \mathrm{sec} / \mathrm{Hz}$ and $12.8 \mathrm{bits} / \mathrm{sec} / \mathrm{Hz}$ for both centralized and distributed resource allocation approaches respectively as in Fig. 6 . Finally, the capacity for the macro UE is measured to be $15 \mathrm{bits} / \mathrm{sec} / \mathrm{Hz}$ for the centralized approach and 14 


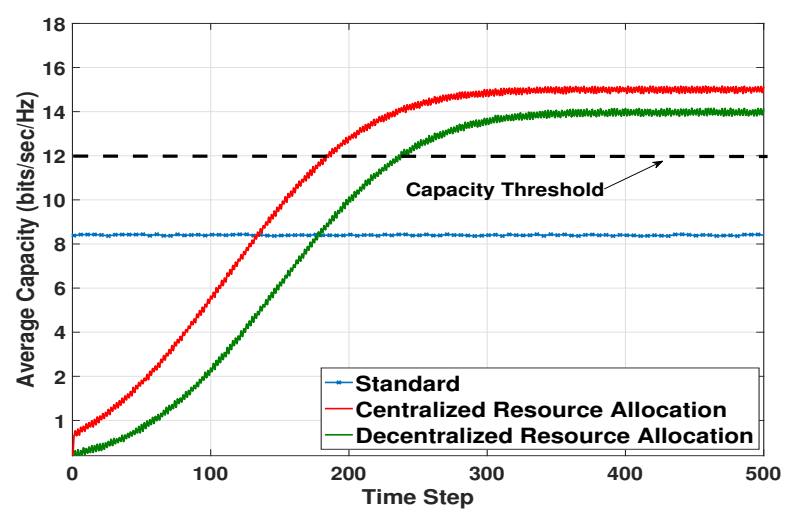

Fig. 7. Average macro BS capacity

bits $/ \mathrm{sec} / \mathrm{Hz}$ for the distributed approach as in Fig. 7 . The convergence time for the centralized scheme is 300 time steps while it is 330 for the decentralized scheme, where the time duration for each step is $0.065 \mathrm{~ms}$.

The BER evaluations for RUE 1, RUE 2 and MUE are plotted in Fig. 8, Fig. 9, and Fig. 10 respectively. The

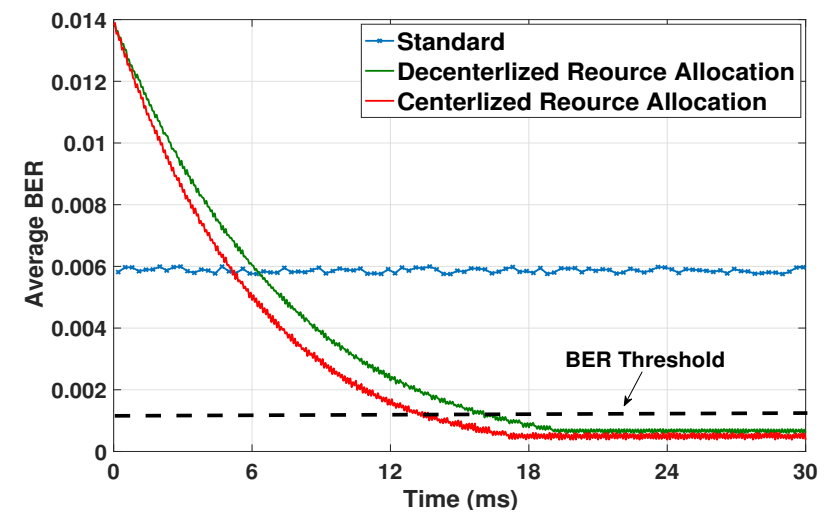

Fig. 8. Average BER experienced by high QoS RUE

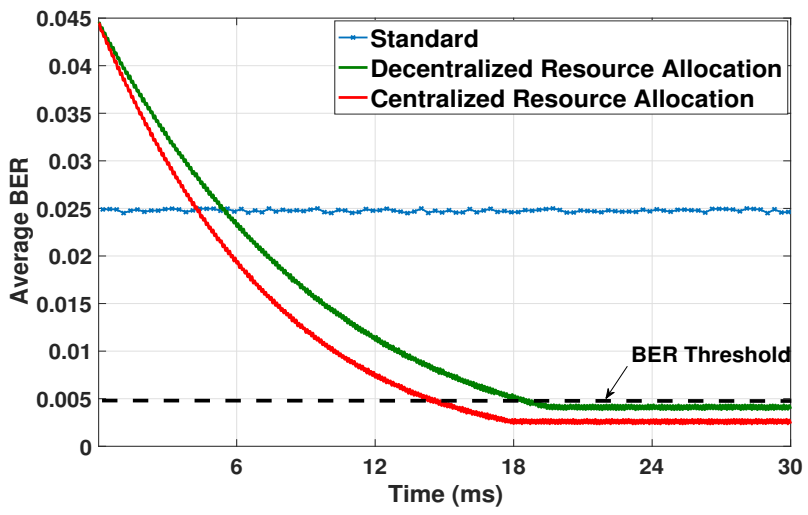

Fig. 9. Average BER experienced by low QoS RUE

proposed resource allocation scheme in both approaches succeeded to maintain BER below the BER threshold which is 0.001 for RUE 1 as in Fig. 8 and below BER threshold for RUE 2 which is 0.005 as in Fig. 9. In addition, the recorded BER for the MUE using both

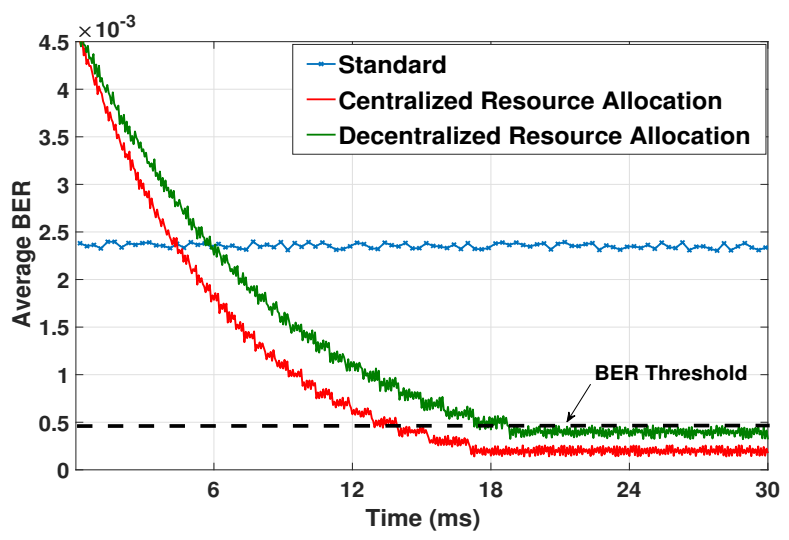

Fig. 10. Average BER experienced by MUE

decentralized and centralized approaches for resource allocation is maintained at 0.00038 and 0.00022 respectively as in Fig. 10. Thus, our scheme reduces the BER by a factor of 10 compared to the standard approach.

The average energy efficiency for the small cell that supports RUE 1 and the small cell that supports RUE 2 are plotted in Fig. 11 and Fig. 12 respectively as a function of time step with duration of $0.065 \mathrm{~ms}$.

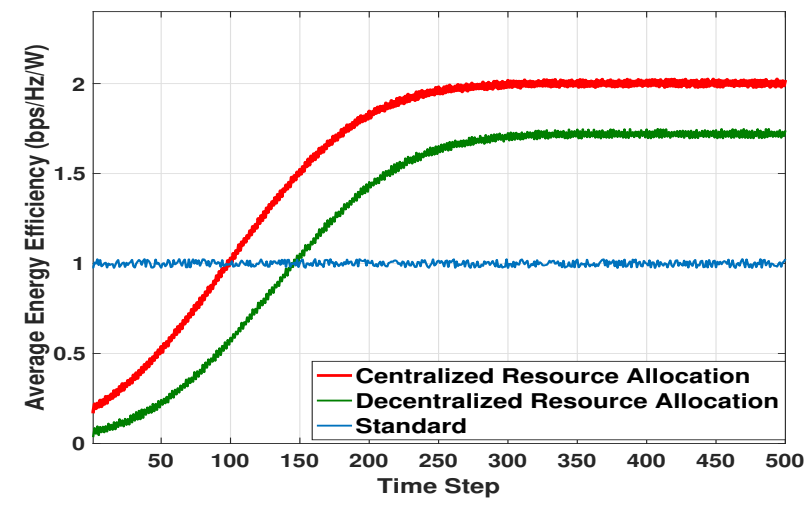

Fig. 11. Average energy efficiency for high QoS pico BS

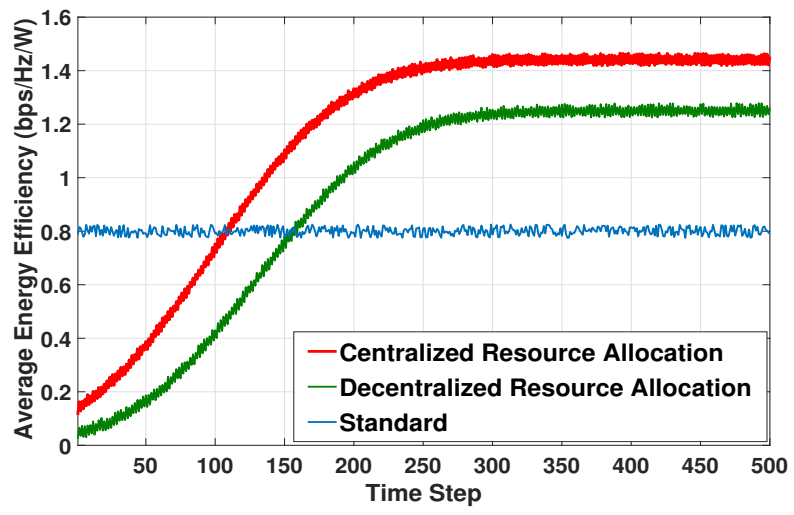

Fig. 12. Average energy efficiency for low QoS pico BS

Fig. 11 shows that our proposed scheme achieves efficiency of $1.9 \mathrm{bps} / \mathrm{Hz} / \mathrm{W}$ and $1.75 \mathrm{bps} / \mathrm{Hz} / \mathrm{W}$ using the centralized and decentralized approaches compared 
to $0.98 \mathrm{bps} / \mathrm{Hz} / \mathrm{W}$ achieved by the standard. On the other hand, our scheme achieves energy efficiency of 1.45 $\mathrm{bps} / \mathrm{Hz} / \mathrm{W}$ and $1.33 \mathrm{bps} / \mathrm{Hz} / \mathrm{W}$ in both centralized and decentralized approaches respectively for RUE 2. Fig. 11 and Fig. 12 also note the convergence time for both approaches, which is 300 for the centralized and 330 for the distributed.

The system evaluation in Fig. 5, to Fig. 12 demonstrates the capability of the implemented sophisticated online learning in resource allocation reflected by the superior results recorded. Moreover, we notice that the proposed scheme converges within short time thanks to the approximation of online learning Q-value that reduces the difference between the approximated Qvalue and the optimal one. Another point to report from the evaluation is that the decentralized approach converges at rate close to the centralized approach. This is because of the conjecture concept exploited in the decentralized learning as in Algorithm 2, which does not require explicit information exchange between the macro BSs and relies on estimation of other BSs action based on the historical knowledge for similar network states. This highlights a significant potential for deploying $\mathrm{H}$ CRANs with distributed resource allocation approach in spite of the little loss in the achieved performance compared to the centralized approach. This is because the centralized approach requires a dedicated controller for resource allocation, which increases the complexity of the network topology and makes it subjected to sudden failure if the controller is down.

\section{Numerical Results}

In this section, we verify the performance of the proposed scheme in terms of energy efficiency, spectral efficiency, and QoS. The evaluation environment consists of three macro BSs with 21 MUEs, 15 RRHs with 47 RUEs accessing $\Gamma_{1}$ and 28 RUEs sharing the spectrum with MUEs in $\Gamma_{2}$. It is assumed that the path-loss model is expressed as $31.5+40 * \log _{10}(d)$ for RRH to RUE link and $31.5+35 * \log _{10}(d)$ for macro BS to RUE and RRH to MUE links, where $d$ is the distance between the transmitter and receiver. Fast-fading coefficients are all generated as independent and identically distributed Rayleigh random variables with unit variances. The data rate thresholds per both types of RUEs and MUE $\theta, \theta^{*}$ and $\delta$ are assumed to be $2 \mathrm{Mbps}, 512 \mathrm{Kbps}$, and $1.2 \mathrm{Mbps}$, respectively. We assume that the number of $\mathrm{BFs}$ is equal to the number of RBs that are within the corresponding sub-band. The rest of the simulation parameters are presented in Table 2. The evaluation of the proposed scheme including energy efficiency, spectral efficiency, and data rate is investigated in the following sections.

\subsection{Energy Efficiency Evaluation}

In this evaluation, we are interested in measuring the speed of convergence and the achieved energy efficiency

\begin{tabular}{ll}
\hline Parameter & Value \\
\hline \hline User distribution & uniform \\
Number of RBs & 50 \\
Total bandwidth & $10 \mathrm{MHz}$ \\
Ratio of $\Gamma_{1}$ and $\Gamma_{2}$ & 0.6 and 0.4 \\
Thermal noise power & $-112 \mathrm{dBm}$ \\
Macro BS transmission power & $43 \mathrm{dBm}$ \\
Back-haul power consumption $P_{b h}$ & $23 \mathrm{dBm}$ \\
Antenna gain for macro/RRH & $17 / 6 \mathrm{~dB}$ \\
Pico maximum transmission power & $25 \mathrm{dBm}$ \\
Trials per experiment & 1000 \\
\hline
\end{tabular}

TABLE 2

Simulation System Parameters

for both types of RUEs. Thus, we conduct three simulations to evaluate the system convergence, achieved energy efficiency with variable maximum transmission power of RRHs and energy efficiency against the SINR threshold of MUEs. First, we plot energy efficiency as function of the number of time steps in Fig. 13. The performance of our centralized and decentralized resource allocation is compared to two schemes including the standard with fixed power allocation in which the same power is allocated for all RBs and the scheme proposed in [24], which aims at tackling the energy efficiency problem in H-CRANs denoted by (EE-HCRAN). Moreover, we include typical online learning resource allocation, which is online Q-learning without the enhancement of the brief representation proposed for the Q-value in which they are approximated as a function of much smaller set of variables. Typical online learning is

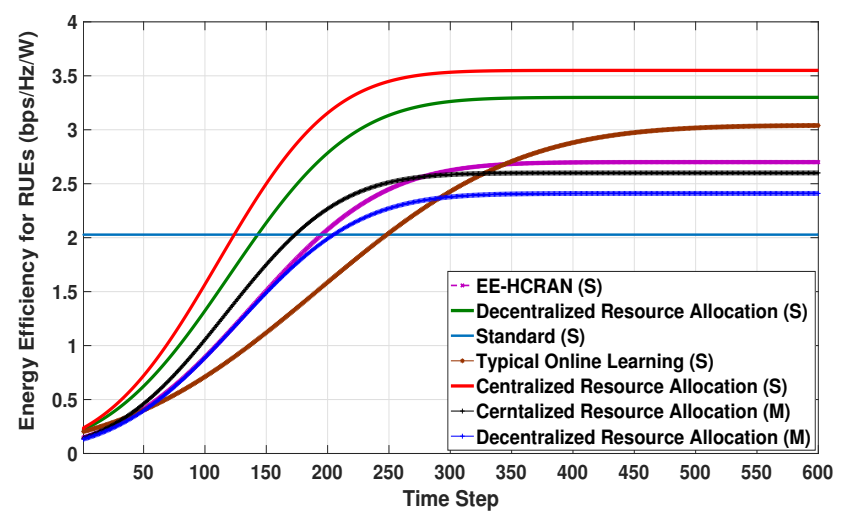

\section{Fig. 13. Energy efficiency convergence}

included to demonstrate the advantage of approximation of online learning in speed of convergence. From Fig. 13, we notice that our scheme converges after 300 iterations faster than typical online learning and EE-HCRAN and achieved the highest energy efficiency.

Second, we plot the achieved energy efficiency against the maximum transmission power of RRH $\left(P_{\max }\right)$ in Fig. 14. It is observed that the average energy efficiency is monotonically non decreasing function of $P_{\max }$. With small value of $P_{\max }$, the energy efficiency increases until it saturates at $P_{\max }$ of $23 \mathrm{dBm}$. This is due to the fact that the compared schemes aim to balance the system energy efficiency and the power consumption. The further increase in transmission power will result 
in degradation in energy efficiency. We notice that both versions of our scheme achieved higher energy efficiency than EE-HCRAN.

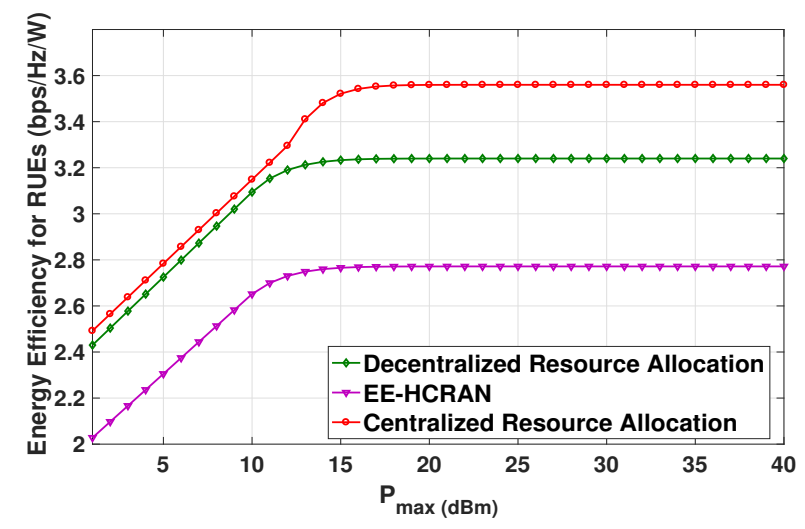

Fig. 14. Energy efficiency with variable $P_{\max }$

The third evaluation focuses on the system with variable SINR threshold of MUEs. Fig. 15 presents the energy efficiency achieved versus the SINR threshold of MUEs accessing $\Gamma_{2}$ with $P_{\max }=25 \mathrm{dBm}$. We notice that the proposed scheme outperforms both EE-HCRAN and the standard schemes. Fig. 15 reveals that when SINR threshold is not large, the energy efficiency is stable with the increasing threshold because the inter-tier interference is not severe. Moreover, the proposed resource allocation scheme achieves the best performance compared to others. This indicates that the proposed solution mitigates the inter-tier interference and provides higher bit rates for MUEs than the other schemes.

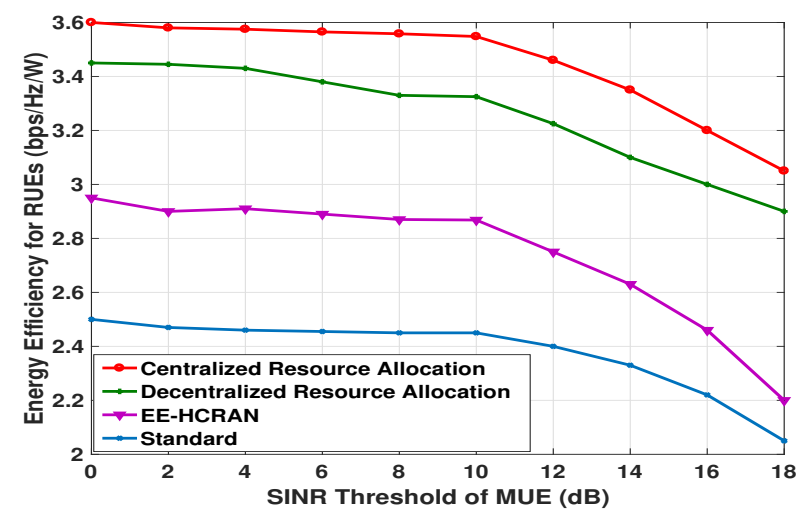

Fig. 15. Energy efficiency with variable SINR threshold for MUEs

\subsection{Spectral Efficiency and QoS Evaluation}

In this section, we evaluate the proposed scheme performance in terms of spectral efficiency and QoS represented by the data rate achieved by both RUEs accessing $\Gamma_{1}$ and $\Gamma_{2}$, and MUEs. Thus, we plot the average system spectral efficiency against the time steps and maximum transmission power of RRH in Fig. 16 and Fig. 17 respectively. Fig. 16 emphasizes the speed of convergence

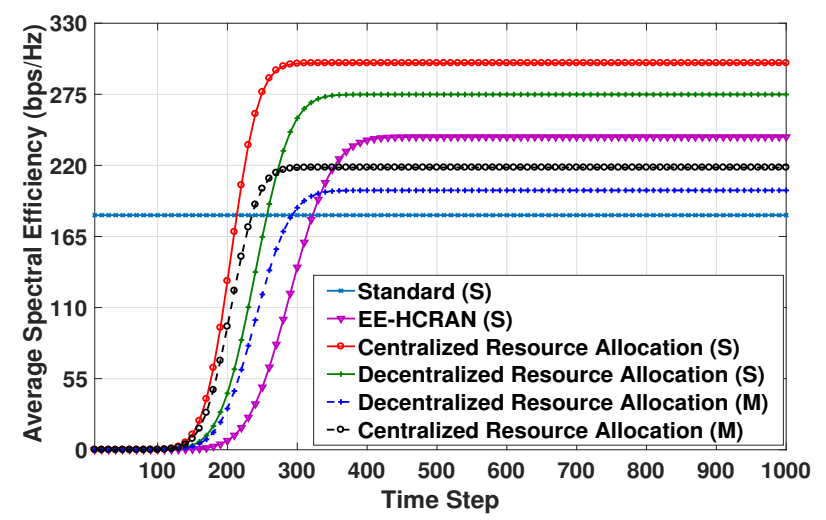

Fig. 16. Spectral efficiency convergence

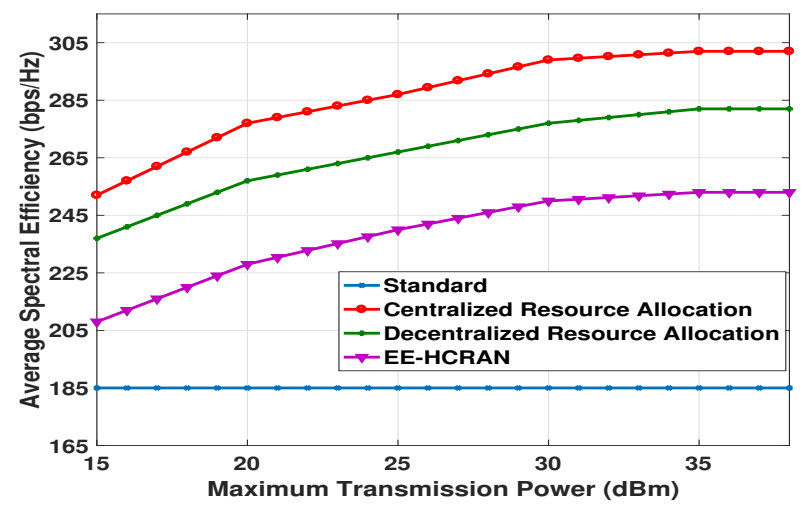

Fig. 17. Spectral efficiency with variable maximum $\mathrm{RRH}$ power

achieved by our scheme compared to others. Our scheme is the fastest with the highest spectral efficiency. In Fig. 17 , spectral efficiency with variable maximum transmission power shows that both approaches of our scheme record the best level of spectral efficiency compared to other schemes.

QoS requirements stated in C2, C3 and C4 are investigated in this evaluation. Fig. 18, Fig. 19 and Fig. 20 present the cumulative distribution function $(\mathrm{CDF})$ of the data rate for both RUEs accessing sub-band $\Gamma_{1}$ and $\Gamma_{2}$, and the CDF of the data rate for MUEs respectively. We notice that our scheme (Centralized RA) and (Decen-

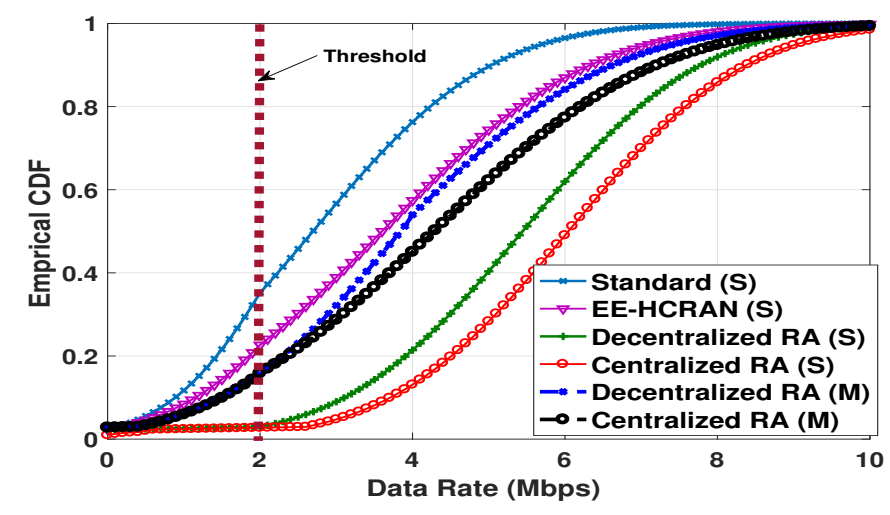

Fig. 18. Data rate CDF for RUEs accessing $\Gamma_{1}$ 
tralized RA) is the only scheme that managed to have more than $97 \%$ of the users above the the specified thresholds $\theta, \theta^{*}$ and $\delta$ compared to EE-HCRAN that records $78 \%$ above threshold, and standard with $65 \%$. This evaluation demonstrates the capability of the online learning scheme to allocate RB and power efficiently while maintaining QoS of users at the maximum level.

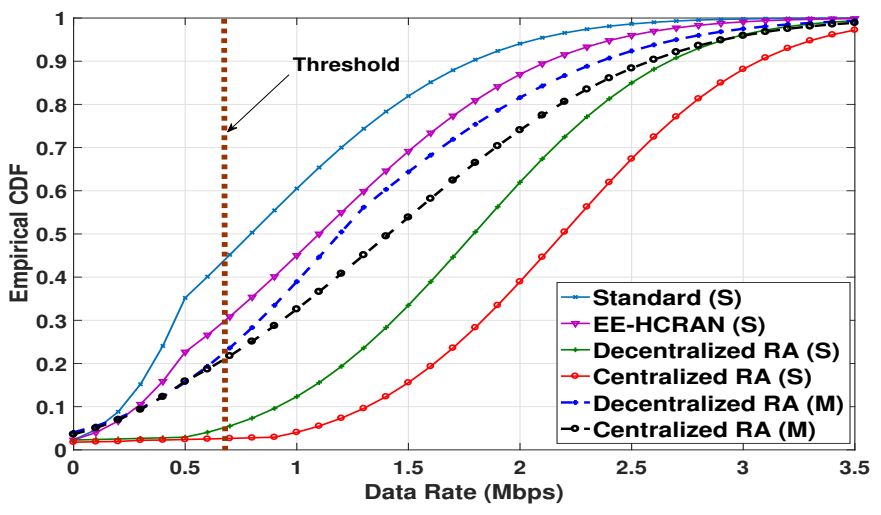

Fig. 19. Data rate CDF for RUEs accessing $\Gamma_{2}$

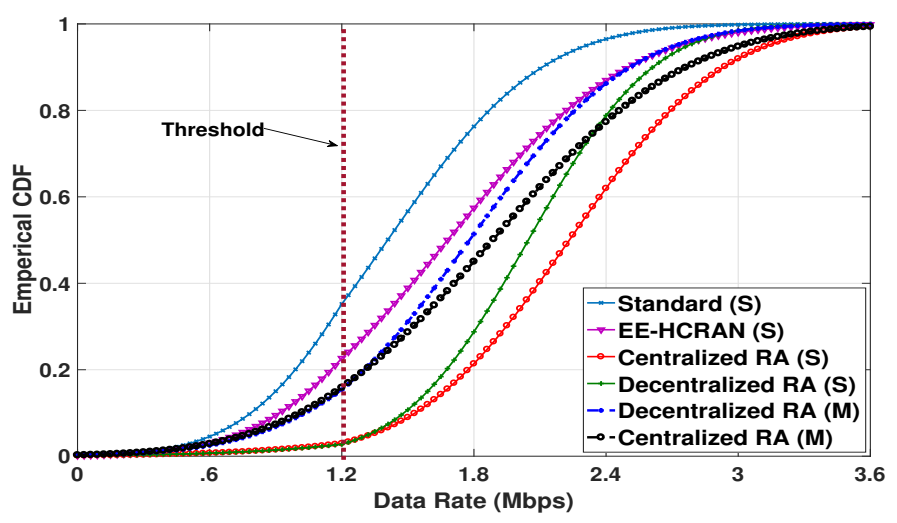

Fig. 20. Data rate CDF for MUEs accessing $\Gamma_{2}$

\subsection{Other Evaluations Aspects and Discussion}

We consider the case of dynamic users who join and leave the system frequently. These users may be viewed as distributed randomly. Their arrival is modeled as homogeneous Poisson point process with intensity $\lambda=3$. Different users have independent duration to stay in the system. We notice the impact of considering users mobility on the achieved energy efficiency, spectral efficiency and data rate in the results of Fig. 13, Fig. 16, and Fig. 18 to Fig. 20. Fig. 13 and Fig. 16 reveal that there is a drop of $27 \%$ in the energy efficiency and spectral efficiency as a result of user mobility when comparing the performance of our scheme in the case of stationary users (S) and mobile users (M). However, our scheme has only a drop of $14 \%$ in the data rate achieved for both types of RUEs and MUEs and all the achieved rates are still above the threshold as in Fig. 18 to Fig. 20. The degradation of the performance in the mobile users case is due to the difficulty to acquire the state information as it changes frequently.

From the presented results, we notice that our proposed scheme with both approaches outperforms the EEHCRAN scheme in terms of the achieved energy efficiency with gain of $16 \%$ for the decentralized resource allocation and $24 \%$ for the centralized resource allocation. The use of machine learning in resource allocation is superior compared to the simple convex optimization used in EE-HCRAN for resource allocation as online learning does not require specific model of the network environment. This is a significant factor as 5G H-CRANs are dynamic, which cannot be tied to specific model. Therefore, online learning, which adopts learning from experience approach is a good fit for the resource allocation problem. In addition, the compact representation for states and approximation for Q-value contribute to the enhancement of speed of convergence in our scheme. Another issue to note in the proposed scheme is that the decentralized approach achieved comparable results in the evaluation, which reflects the advantage of the conjecture feature in the exploited learning approach. This feature eliminates the need for explicit cooperation between the macro BSs to exchange information, which improves the quality of the selected action. In addition, this relieves the BBU pool from the resource allocation task and reduces the signal processing overhead. Another factor for the superior performance achieved is the sophisticated RB allocation mechanism that considers both location and QoS of the RUEs in addition to the appropriate power allocation, which has a considerable contribution to the achieved results.

Furthermore, we compare the testbed results and numerical results in terms of energy efficiency for high QoS RUEs, energy efficiency for low QoS RUEs, system spectral efficiency, data rate for both types RUEs and MUEs, and convergence speed. The results are compared on the basis of the optimal (maximum) value reached for energy efficiency and spectral efficiency and on average for data rate. Table 3 presents the comparison results. We notice that the numerical results record better values

\begin{tabular}{lllll}
\hline Evaluation Metric & $\begin{array}{l}\text { Proposed } \\
\text { Centralized } \\
\text { Testbed }\end{array}$ & $\begin{array}{l}\text { Proposed } \\
\text { Decentralized } \\
\text { Testbed }\end{array}$ & $\begin{array}{l}\text { Proposed } \\
\text { Centralized } \\
\text { (S) } \\
\text { Simulation }\end{array}$ & $\begin{array}{l}\text { Proposed } \\
\text { Decentralized } \\
\text { (S) }\end{array}$ \\
\hline \hline $\begin{array}{l}\text { Simulation } \\
\text { for high QoS RUEs }\end{array}$ & $\begin{array}{l}2 \\
\mathrm{bps} / \mathrm{Hz} / \mathrm{W}\end{array}$ & $\begin{array}{l}1.7 \\
\mathrm{bps} / \mathrm{Hz} / \mathrm{W}\end{array}$ & $\begin{array}{l}2.15 \\
\mathrm{bps} / \mathrm{Hz} / \mathrm{W}\end{array}$ & $\begin{array}{l}1.92 \\
\mathrm{bps} / \mathrm{Hz} / \mathrm{W}\end{array}$ \\
$\begin{array}{l}\text { Energy efficiency } \\
\text { for low QoS RUEs }\end{array}$ & $\begin{array}{l}1.45 \\
\mathrm{bps} / \mathrm{Hz} / \mathrm{W}\end{array}$ & $\begin{array}{l}1.25 \\
\mathrm{bps} / \mathrm{Hz} / \mathrm{W}\end{array}$ & $\begin{array}{l}1.62 \\
\mathrm{bps} / \mathrm{Hz} / \mathrm{W}\end{array}$ & $\begin{array}{l}1.34 \\
\mathrm{bps} / \mathrm{Hz} / \mathrm{W}\end{array}$ \\
\hline Spectral efficiency & $285 \mathrm{bps} / \mathrm{Hz}$ & $260 \mathrm{bps} / \mathrm{Hz}$ & $302 \mathrm{bps} / \mathrm{Hz}$ & $275 \mathrm{bps} / \mathrm{Hz}$ \\
\hline $\begin{array}{l}\text { Average data rate } \\
\text { for high QoS RUEs }\end{array}$ & $5.6 \mathrm{Mbps}$ & $5 \mathrm{Mbps}$ & $6 \mathrm{Mbps}$ & $5.55 \mathrm{Mbps}$ \\
$\begin{array}{l}\text { Average data rate } \\
\text { for low QoS RUEs }\end{array}$ & $2 \mathrm{Mbps}$ & $1.6 \mathrm{Mbps}$ & $2.2 \mathrm{Mbps}$ & $1.78 \mathrm{Mbps}$ \\
$\begin{array}{l}\text { Average data rate } \\
\text { for MUEs }\end{array}$ & $2.1 \mathrm{Mbps}$ & $1.73 \mathrm{Mbps}$ & $2.3 \mathrm{Mbps}$ & $1.9 \mathrm{Mbps}$ \\
\hline $\begin{array}{l}\text { Convergence Speed } \\
\text { (time steps) }\end{array}$ & 300 & & & \\
\hline \hline
\end{tabular}

TABLE 3

Testbed and numerical results comparison 
than the testbed implementation. The reason for that little win is due to real time interfering means such as other wireless devices in the lab. In addition, hardware limitation on processing and synchronization with the controlling unit may degrade the achieved results.

Finally, we study the impact of the spectrum partitioning on the achieved energy efficiency and spectral efficiency. This impact is evaluated in Fig. 21 and Fig. 22 respectively, with assumption that each RUE is allocated at least one RB to guarantee minimum QoS requirements. Fig. 21 and Fig. 22 show that the energy efficiency and spectral efficiency increase linearly with the ratio of $\Gamma_{1}$ to $\Gamma_{A}\left(\Gamma_{A}=\right.$ the total RBs for both $\Gamma_{1}$ and $\left.\Gamma_{2}\right)$. Moreover, the centralized resource allocation achieves better efficiency than the decentralized approach. Increasing the ratio $\Gamma_{1}$ to $\Gamma_{A}$ against $\Gamma_{2}$ provides more RBs for RRHs, reduces the inter-tier interference as the shared RBs in $\Gamma_{2}$ are decreased. In addition, the increase of $\Gamma_{1}$ ratio enhances the achieved SINR for RUEs and it allows the RUEs that access $\Gamma_{2}$ to raise their transmission power as the inter-tier interference is reduced. This will further enhances the energy efficiency of the H-CRANs. Despite the fact that the increase of $\Gamma_{1}$ enhances the achieved energy and spectral efficiencies, it affects the fairness of resource allocation, which opens directions for future research on how to balance this tradeoff.

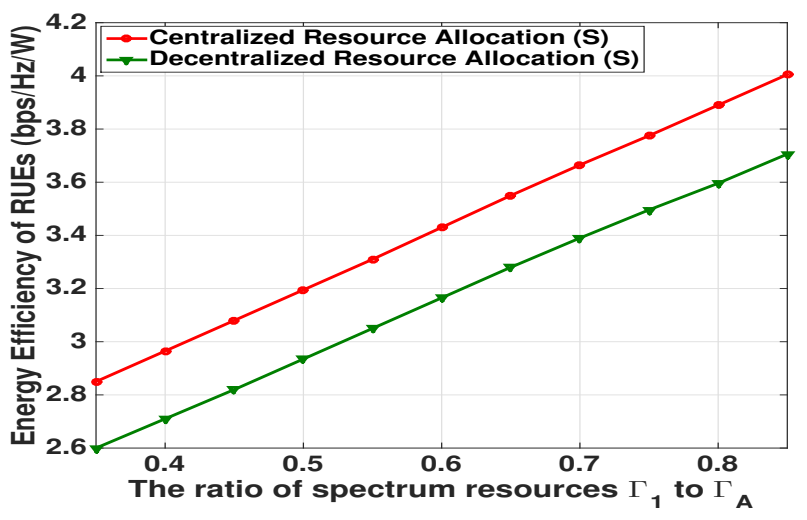

Fig. 21. Energy Efficiency for different ratios of $\Gamma_{1}$ to $\Gamma_{A}$

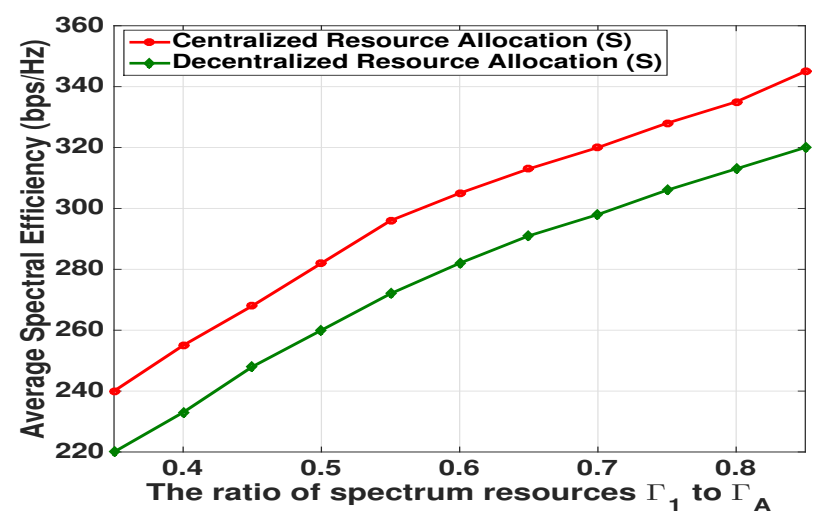

Fig. 22. Spectral Efficiency for different ratios of $\Gamma_{1}$ to $\Gamma_{A}$

\section{Conclusion}

In this paper, green resource allocation scheme in $\mathrm{H}$ CRANs following online learning based centralized and decentralized approaches is proposed. RBs and transmission power are allocated subjected to inter-tier interference and capacity constraints. The centralized approach places a dedicated controller integrated to the BBU pool to perform resource allocation while the decentralized approach seizes the macro BSs awareness about RRHs operates under their coverage and assign them the task of resource allocation in a distributed fashion. Sophisticated frequency partitioning is proposed to account for interference and support better online learning exploration. Approximated online learning methodology is established to perform joint allocation for RB and transmission power. Testbed implementations and simulation results including energy efficiency, spectral efficiency, BER evaluation and data rate have demonstrated the capability of the proposed scheme to allocate resources in green and suppressed interference fashion.

\section{REFERENCES}

[1] M. Agiwal, A. Roy, and N. Saxena, "Next generation $5 g$ wireless networks: A comprehensive survey", IEEE Communications Surveys Tutorials, vol. 18, no. 3, pp. 1617-1655, thirdquarter 2016.

[2] Nisha Panwar, Shantanu Sharma, and Awadhesh Kumar Singh, "A survey on 5g: The next generation of mobile communication", Physical Communication, vol. 18, Part 2, pp. 64 - 84, 2016, Special Issue on Radio Access Network Architectures and Resource Management for $5 \mathrm{G}$.

[3] C. L. I, C. Rowell, S. Han, Z. Xu, G. Li, and Z. Pan, “Toward green and soft: a 5 g perspective", IEEE Communications Magazine, vol. 52, no. 2, pp. 66-73, February 2014.

[4] M. Peng and W. Wang, "Technologies and standards fortd-scdma evolutions to imt-advanced", IEEE Communications Magazine, vol. 47, no. 12, pp. 50-58, Dec 2009.

[5] Y. Cai, F. R. Yu, and S. Bu, "Dynamic operations of cloud radio access networks (c-ran) for mobile cloud computing systems", IEEE Transactions on Vehicular Technology, vol. 65, no. 3, pp. 15361548, March 2016.

[6] M. Peng, C. Wang, V. Lau, and H. V. Poor, "Fronthaul-constrained cloud radio access networks: insights and challenges", IEEE Wireless Communications, vol. 22, no. 2, pp. 152-160, April 2015.

[7] Y. Shi, J. Zhang, and K. B. Letaief, "Group sparse beamforming for green cloud-ran", IEEE Transactions on Wireless Communications, vol. 13, no. 5, pp. 2809-2823, May 2014.

[8] Y. Cai, F. R. Yu, and S. Bu, "Dynamic operations of cloud radio access networks (c-ran) for mobile cloud computing systems", IEEE Transactions on Vehicular Technology, vol. 65, no. 3, pp. 15361548, March 2016

[9] D. W. K. Ng and R. Schober, "Secure and green swipt in distributed antenna networks with limited backhaul capacity", IEEE Transactions on Wireless Communications, vol. 14, no. 9, pp. 5082-5097, Sept 2015.

[10] M. Peng, Y. Li, T. Q. S. Quek, and C. Wang, "Device-to-device underlaid cellular networks under rician fading channels", IEEE Transactions on Wireless Communications, vol. 13, no. 8, pp. 42474259, Aug 2014.

[11] M. Peng, D. Liang, Y. Wei, J. Li, and H. H. Chen, "Selfconfiguration and self-optimization in lte-advanced heterogeneous networks", IEEE Communications Magazine, vol. 51, no. 5, pp. 36-45, May 2013.

[12] R. Q. Hu, Y. Qian, S. Kota, and G. Giambene, "Hetnets - a new paradigm for increasing cellular capacity and coverage [guest editorial]", IEEE Wireless Communications, vol. 18, no. 3, pp. 89, June 2011. 
[13] S. Sun, K. Adachi, P. H. Tan, Y. Zhou, J. Joung, and C. K. Ho, "Heterogeneous network: An evolutionary path to 5g", in 2015 21st Asia-Pacific Conference on Communications (APCC), Oct 2015, pp. $174-178$.

[14] Y. Dong, Z. Chen, P. Fan, and K. B. Letaief, "Mobility-aware uplink interference model for $5 \mathrm{~g}$ heterogeneous networks", IEEE Transactions on Wireless Communications, vol. 15, no. 3, pp. 22312244, March 2016.

[15] Tao Han, Guoqiang Mao, Qiang Li, Lijun Wang, and Jing Zhang, "Interference minimization in $5 \mathrm{~g}$ heterogeneous networks", Mobile Networks and Applications, vol. 20, no. 6, pp. 756-762, 2015.

[16] Z. Fei, C. Xing, N. Li, and J. Kuang, "Adaptive multiobjective optimisation for energy efficient interference coordination in multicell networks", IET Communications, vol. 8, no. 8, pp. 1374-1383, May 2014.

[17] C. Y. Ho and C. Y. Huang, "Energy efficient subcarrier-power allocation and relay selection scheme for ofdma-based cooperative relay networks", in 2011 IEEE International Conference on Communications (ICC), June 2011, pp. 1-6.

[18] Y. Wang, W. Xu, K. Yang, and J. Lin, “Optimal energy-efficient power allocation for ofdm-based cognitive radio networks", IEEE Communications Letters, vol. 16, no. 9, pp. 1420-1423, September 2012.

[19] R. Q. Hu and Y. Qian, "An energy efficient and spectrum efficient wireless heterogeneous network framework for $5 \mathrm{~g}$ systems", IEEE Communications Magazine, vol. 52, no. 5, pp. 94-101, May 2014.

[20] I. AlQerm and B. Shihada, "A cooperative online learning scheme for resource allocation in $5 \mathrm{~g}$ systems", in 2016 IEEE International Conference on Communications (ICC), May 2016, pp. 1-7.

[21] M. Peng, Y. Li, J. Jiang, J. Li, and C. Wang, "Heterogeneous cloud radio access networks: a new perspective for enhancing spectral and energy efficiencies", IEEE Wireless Communications, vol. 21, no. 6, pp. 126-135, December 2014.

[22] M. A. Marotta, N. Kaminski, I. Gomez-Miguelez, L. Z. Granville, J. Rochol, L. DaSilva, and C. B. Both, "Resource sharing in heterogeneous cloud radio access networks", IEEE Wireless Communications, vol. 22, no. 3, pp. 74-82, June 2015.

[23] D. Lopez-Perez, X. Chu, A. V. Vasilakos, and H. Claussen, "Power minimization based resource allocation for interference mitigation in ofdma femtocell networks", IEEE Journal on Selected Areas in Communications, vol. 32, no. 2, pp. 333-344, February 2014.

[24] M. Peng, K. Zhang, J. Jiang, J. Wang, and W. Wang, "Energyefficient resource assignment and power allocation in heterogeneous cloud radio access networks", IEEE Transactions on Vehicular Technology, vol. 64, no. 11, pp. 5275-5287, Nov 2015.

[25] X. Sun and S. Wang, "Resource allocation scheme for energy saving in heterogeneous networks", IEEE Transactions on Wireless Communications, vol. 14, no. 8, pp. 4407-4416, Aug 2015.

[26] J. Tang, D. K. C. So, E. Alsusa, K. A. Hamdi, and A. Shojaeifard, "Resource allocation for energy efficiency optimization in heterogeneous networks", IEEE Journal on Selected Areas in Communications, vol. 33, no. 10, pp. 2104-2117, Oct 2015.

[27] W. Pramudito and E. Alsusa, "A hybrid resource management technique for energy and qos optimization in fractional frequency reuse based cellular networks", IEEE Transactions on Communications, vol. 61, no. 12, pp. 4948-4960, December 2013.

[28] Y. Zhang, Y. Wang, and W. Zhang, "Energy efficient resource allocation for heterogeneous cloud radio access networks with user cooperation and qos guarantees", in 2016 IEEE Wireless Communications and Networking Conference, April 2016, pp. 1-6.

[29] N. Saquib, E. Hossain, and D. I. Kim, "Fractional frequency reuse for interference management in lte-advanced hetnets", IEEE Wireless Communications, vol. 20, no. 2, pp. 113-122, April 2013.

[30] D. Feng, C. Jiang, G. Lim, L. J. Cimini, G. Feng, and G. Y. Li, "A survey of energy-efficient wireless communications", IEEE Communications Surveys Tutorials, vol. 15, no. 1, pp. 167-178, First 2013.

[31] Richard S. Sutton and Andrew G. Barto, Introduction to Reinforcement Learning, MIT Press, Cambridge, MA, USA, 1st edition, 1998.

[32] C. J. C. H. Watkins and P. Dayan, "Q-learning", Mach. Learn, vol. 8, no. 3, pp. 279-292, 1992

[33] X. Zhou, R. Zhang, and C. K. Ho, "Wireless information and power transfer: Architecture design and rate-energy tradeoff", IEEE Transactions on Communications, vol. 61, no. 11, pp. 47544767, November 2013.

[34] A. D. Tijsma, M. M. Drugan, and M. A. Wiering, "Comparing exploration strategies for q-learning in random stochastic mazes", in 2016 IEEE Symposium Series on Computational Intelligence (SSCI), Dec 2016, pp. 1-8.

[35] A. Checko, H. L. Christiansen, Y. Yan, L. Scolari, G. Kardaras, M. S. Berger, and L. Dittmann, "Cloud ran for mobile networks;a technology overview", IEEE Communications Surveys Tutorials, vol. 17, no. 1, pp. 405-426, Firstquarter 2015.

[36] Eyal Even-Dar and Yishay Mansour, "Convergence of optimistic and incremental q-learning", in Proceedings of the 14th International Conference on Neural Information Processing Systems: Natural and Synthetic, Cambridge, MA, USA, 2001, NIPS'01, pp. 1499-1506, MIT Press.

[37] Vassilis A. Papavassiliou and Stuart Russell, "Convergence of reinforcement learning with general function approximators", in Proceedings of the 16th International Joint Conference on Artificial Intelligence - Volume 2, San Francisco, CA, USA, 1999, IJCAI'99, pp. 748-755, Morgan Kaufmann Publishers Inc.

[38] "On nonlinear fractional programming", Management Science, vol. 13, no. 7, pp. 492-498, 1967

[39] Gareth O. Roberts and Jeffrey S. Rosenthal, "Harris recurrence of metropolis-within-gibbs and trans-dimensional markov chains", The Annals of Applied Probability, vol. 16, no. 4, pp. 2123-2139, 2006.

[40] L. N. de Castro and F. J. Von Zuben, "Learning and optimization using the clonal selection principle", IEEE Transactions on Evolutionary Computation, vol. 6, no. 3, pp. 239-251, Jun 2002.

[41] Y. S. Soh, T. Q. S. Quek, M. Kountouris, and H. Shin, "Energy efficient heterogeneous cellular networks", IEEE Journal on Selected Areas in Communications, vol. 31, no. 5, pp. 840-850, May 2013.

[42] Francesca Gagliardi, "Institutions and economic change: A critical survey of the new institutional approaches and empirical evidence", Journal of Behavioral and Experimental Economics (formerly The Journal of Socio-Economics), vol. 37, no. 1, pp. 416-443, February 2008.

[43] GNU Radio, "[online available at]", http://gnuradio.org/trac.

[44] SDR Forum, "Cognitive radio work group", https://www.ettus.com/product/details/UN210-KIT/.

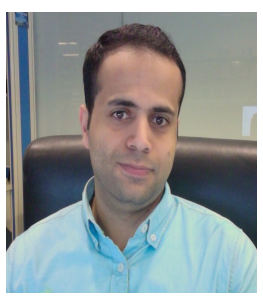

Ismail AIQerm is a $\mathrm{PhD}$ candidate in computer science at King Abdullah University of Science and Technology (KAUST) and a senior member of the networking lab led by Prof. Basem Shihada. Ismail joined King Abdullah University of Science and Technology in 2009 as a founding master student in Electrical Engineering and was among the recipients of KAUST Provost Award. Prior Joining KAUST, he received bachelor of Computer Engineering from King Fahd University of Petroleum and Minerals (KFUPM). His research interests include Cognitive radio, Resource allocation in heterogeneous cellular networks, Developing machine learning techniques for resource allocation in wireless networks, and software defined radio prototypes.

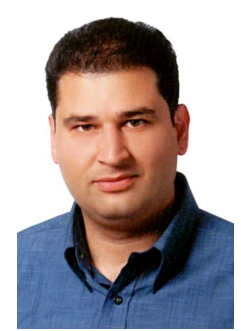

Basem Shihada is an Associate and Founding Professor of computer science and electrical engineering in the Computer, Electrical and Mathematical Sciences and Engineering (CEMSE) Division at King Abdullah University of Science and Technology (KAUST). Before joining KAUST in 2009, he was a visiting faculty at the Computer Science Department in Stanford University. His current research covers a range of topics in energy and resource allocation in wired and wireless communication networks, including wireless mesh, wireless sensor, multimedia, and optical networks. He is also interested in SDNs, IoT, and cloud computing. In 2012, he was elevated to the rank of Senior Member of IEEE. 